\title{
Efficient and practical syntheses of mannose tri-, tetra-, penta-, hexa-, hepta-, and octasaccharides existing in $\mathbf{N}$-glycans
}

\author{
Jianjun Zhang and Fanzuo Kong* \\ Research Center for Eco-Environmental Sciences, Chinese Academy of Sciences, PO Box 2871, Beijing 100085, China
}

Received 16 January 2001; accepted 21 February 2002

Abstract-An efficient and practical regio- and stereoselective synthesis of mannose tri-, tetra-, penta-, hexa-, hepta-, and octasaccharides of $N$-glycans was achieved using simple mannosyl derivatives as the starting materials. (C) 2002 Elsevier Science Ltd. All rights reserved.

\section{Introduction}

$N$-Linked glycans play a vital role in fundamental biological processes such as cell differentiation, malignant transformation, viral, bacterial and parasitic infections and protein transportations. ${ }^{1}$ Recent studies have revealed that the mannose oligosaccharides in $N$-glycans are required for human CD2 adhesion function ${ }^{2}$ and high mannose oligosaccharides are related to HIV infection. ${ }^{3}$ For a study of the details of the recognition mechanism, chemically synthesized oligosaccharides are required because such homogeneous oligosaccharide samples are obtainable in only very small amounts from natural sources. Remarkable progress has been made in the field of $\mathrm{N}$-glycan synthesis, ${ }^{4}$ however, multiple steps and orthogonal masking groups are needed in most of the syntheses making the work complex and time consuming. We present herein a very efficient and practical method for the synthesis of mannose oligosaccharides (Man3, Man4, Man5, Man6, Man7, and Man8) for $N$-glycans, ${ }^{5}$ as shown in Scheme 1.

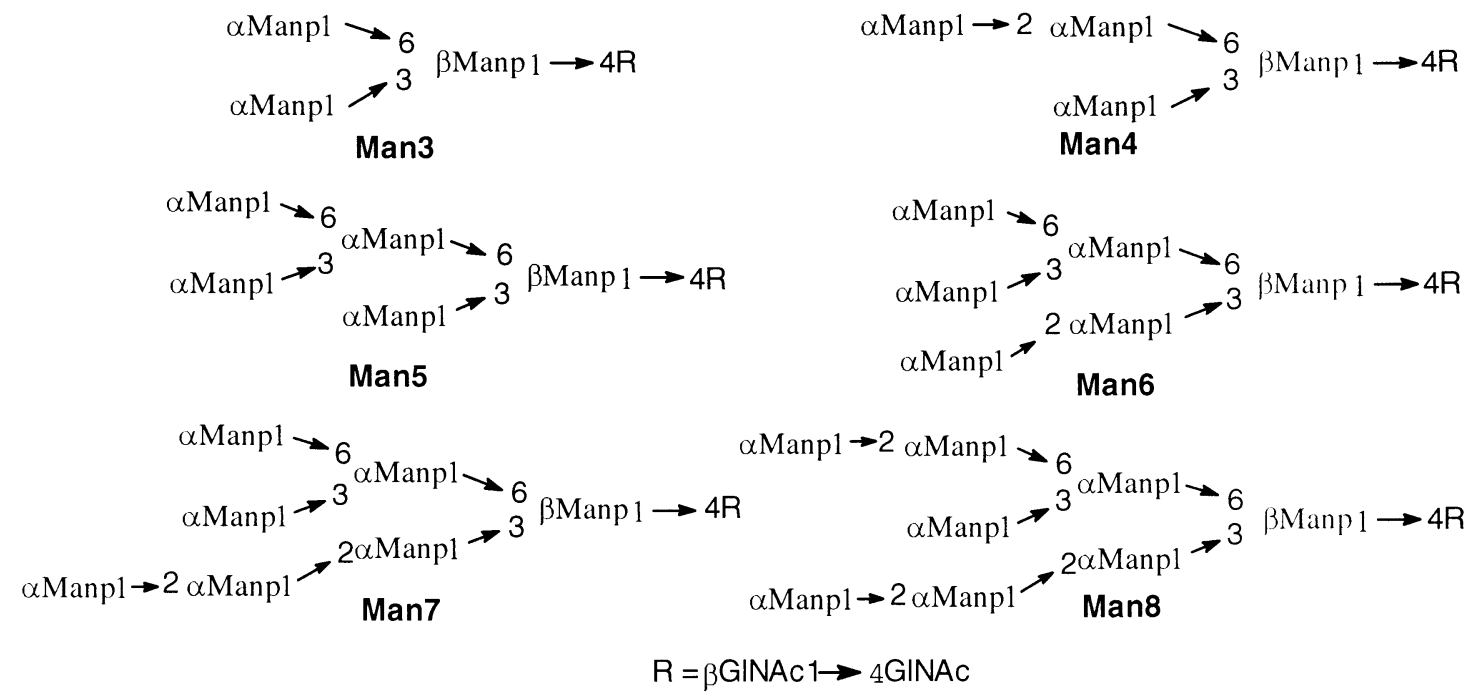

Scheme 1.

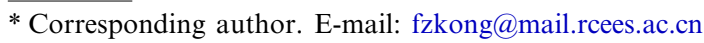




\section{Results and discussion}

Previous syntheses of mannose oligosaccharides involved the use of permanent and temporary protecting groups as well as complex protection and deprotection procedures. ${ }^{4}$ Thus, it is difficult to obtain the oligosaccharide samples in sufficient quantity through these syntheses. In the work described here we designed a new method for mannose oligosaccharide syntheses using solely temporary protecting groups in combina- tion with regioselective glycosylation and one-pot multistep transformation protocols.

Scheme 2 depicts the syntheses of Man3, Man4, and Man 5, and Scheme 3 shows the syntheses of Man6, Man7, and Man8. In our synthetic route, the target oligosaccharides were constructed from the coupling of fully acylated mannose mono- and oligosaccharide trichloroacetimidate donors with the mannose di-, tri-, and terasaccharide acceptors having free 4,6-hydroxyl
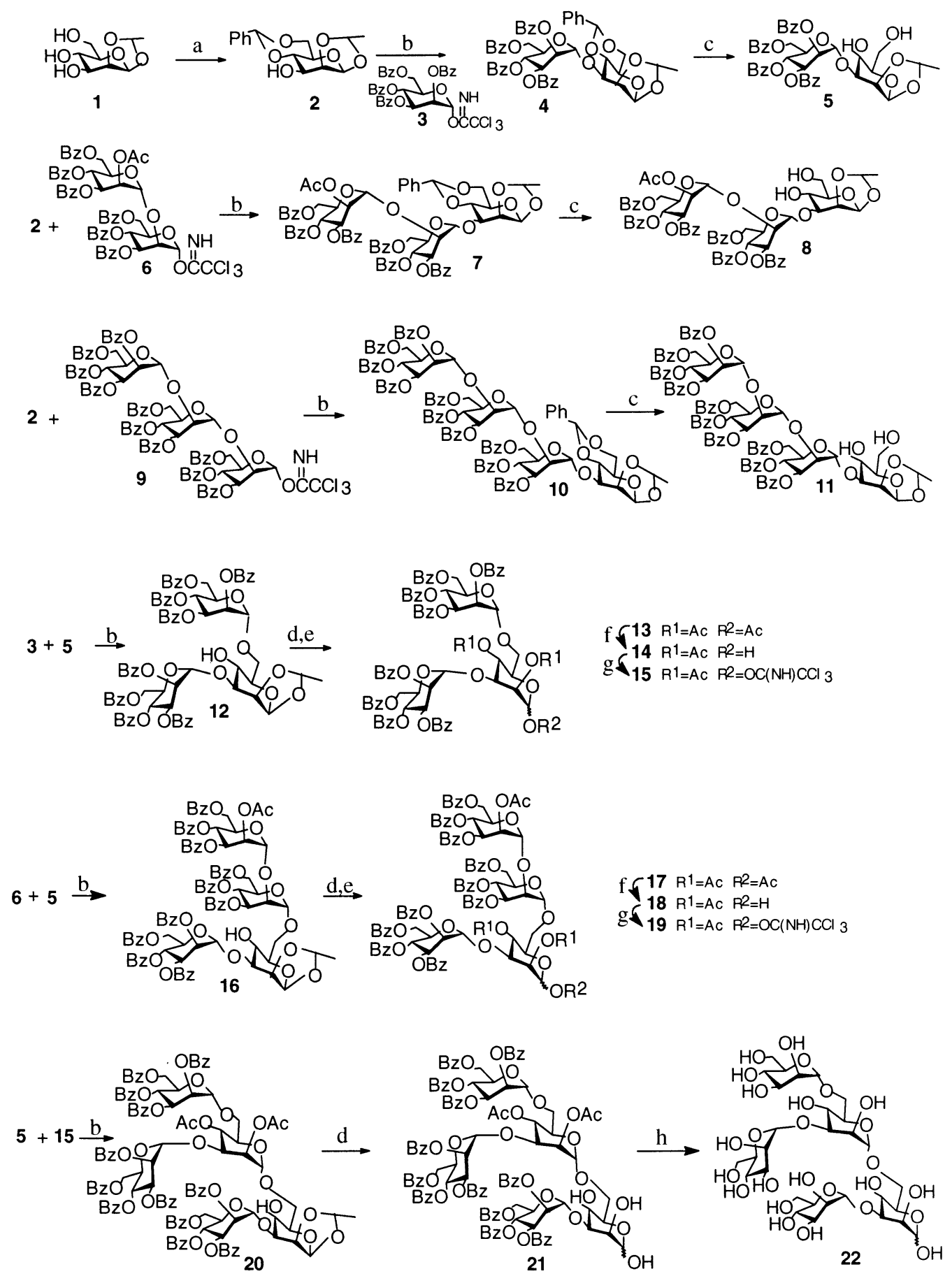

Scheme 2. Reagents and conditions: (a) $\mathrm{PhCHO}, \mathrm{HC}(\mathrm{OEt})_{3}$, toluenesulfonic acid, rt, $12 \mathrm{~h}(88 \%)$; (b) $\mathrm{TMSOTf} \mathrm{CH}_{2} \mathrm{Cl}_{2}, 0^{\circ} \mathrm{C}, 2 \mathrm{~h}$; (c) $0.1 \% \mathrm{HCl}-\mathrm{MeOH}, \mathrm{rt}, 12 \mathrm{~h}\left(88 \%\right.$ ); (d) $90 \% \mathrm{CF}_{3} \mathrm{COOH}, \mathrm{rt}, 2-5 \mathrm{~h}$; (e) $\mathrm{Ac}_{2} \mathrm{O} /$ pyridine (dry), rt, 6 h; (f) $1.0 \mathrm{M} \mathrm{NH} / \mathrm{MeOH}, 3 \mathrm{~h}$;

(g) $\mathrm{CCl}_{3} \mathrm{CN}, \mathrm{DBU}, \mathrm{CH}_{2} \mathrm{Cl}_{2} 3 \mathrm{~h}$ (for 15: $82 \%$ from 12; for 19: 82\% from 16); (h) satd $\mathrm{NH}_{3} / \mathrm{MeOH}^{\mathrm{rt},} 7 \mathrm{days}$ (76\%). 

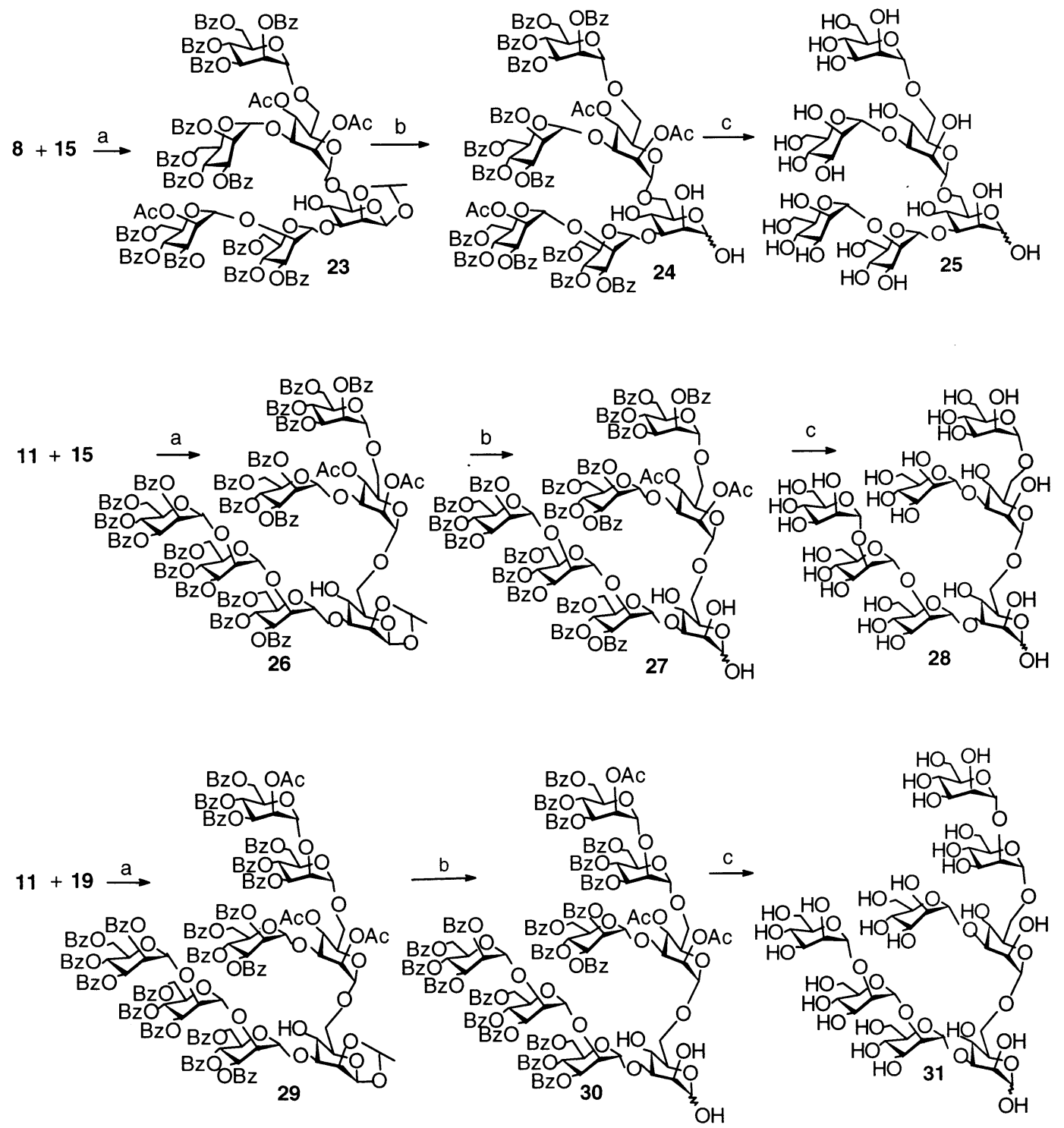

Scheme 3. Reagents and conditions: (a) TMSOTf, $\mathrm{CH}_{2} \mathrm{Cl}_{2}, 0{ }^{\circ} \mathrm{C}, 2 \mathrm{~h}\left(79 \%\right.$ for $\mathbf{2 3}, 82 \%$ for 26, 80\% for 29); (b) $90 \% \mathrm{CF}_{3} \mathrm{COOH}$, rt, 2-5 h (81\% for 24, $87 \%$ for 27, 85\% for 30); (c) satd $\mathrm{NH}_{3} / \mathrm{MeOH}$, rt, 7 days (72\% for 25, 76\% for 28, 82\% for 31).

groups at the reducing end. This strategy ensures good reactivity and selectivity of the couplings because of the primary hydroxyl in the acceptors, ${ }^{6}$ while the preparations of the trichloroacetimidate donors are relatively easy. Thus, 4,6- $O$-benzylidene-1,2- $O$-ethylidene- $\beta$-Dmannopyranose 2 , which was obtained from 1,2-O -ethylidene- $\beta$-D-mannopyranose, ${ }^{7}$ was chosen as the starting material. Condensation of $\mathbf{2}$ with perbenzoylated mannopyransyl trichloroacetimidate $\mathbf{3}$ afforded the disaccharide 4 in high yield (83\%). Selective removal of the benzylidene protecting group with $0.1 \% \mathrm{HCl}-$ $\mathrm{MeOH}$ at room temperature from $\mathbf{4}$ was carried out smoothly, yielding the disaccharide acceptor $5(88 \%)$. Coupling of $\mathbf{2}$ with the disaccharide donor $\mathbf{6}$, which was readily prepared by self condensation of 1,2-O -allyloxyethylidene-3,4,6-tri- $O$-benzoyl- $\beta$-D-mannopyranose followed by deallylation and trichloroacetimidation, ${ }^{8}$ gave 7 in high yield (92\%), and subsequent debenzylidenation gave the trisaccharide acceptor $8(95 \%)$. Coupling of 2 with the trisaccharide donor $\mathbf{9}^{9}(\rightarrow \mathbf{1 0}, 86 \%)$, followed by debenzylidenation furnished the tetrasaccharide acceptor 11 (92\%).

With these acceptors in hand, the required mannosyl oligosaccharides were synthesized readily. Thus, condensation of $\mathbf{5}$ with the monosaccharide donor $\mathbf{3}$ gave the 6-linked trisaccharide $\mathbf{1 2}$ with high selectivity $(87 \%)$. Subsequent hydrolysis to remove the ethylidene protecting group, acetylation with $\mathrm{Ac}_{2} \mathrm{O}$ in pyridine, selective 1-O-deacetylation with dilute $\mathrm{NH}_{3}-\mathrm{MeOH}$ solution, followed by trichloroacetimidation produced the trisaccharide donor 15 (overall yield for the four steps $82 \%$ ). These four steps were carried out consecutively without chromatographic separation for the first three steps, making large scale preparation possible with high yields. The ${ }^{1} \mathrm{H}$ NMR spectrum of $\mathbf{1 5}$ showed three triplets for $\mathrm{C}(4) \mathrm{H}$ of the mannose at $\delta 6.22\left(J_{3,4}=J_{4,5}=\right.$ $9.8 \mathrm{~Hz}), 6.11\left(J_{3,4}=J_{4,5}=9.9 \mathrm{~Hz}\right), 5.51 \mathrm{ppm}\left(J_{3,4}=J_{4,5}=\right.$ $9.8 \mathrm{~Hz}$ ), confirming the 6-selective mannosylation of 5 as the third downfield triplet was obtained through 
acetylation of the upfield $\mathrm{C}(4) \mathrm{H}$ of $\mathbf{1 2}$. The tetrasaccharide donor 19 was prepared in the same way, i.e. coupling of 5 with 6 gave $16(78 \%)$, and subsequent hydrolysis, acetylation, 1-O-deacetylation, and trichloroacetimidation gave 19 (overall yield 84\%). Condensation of the trisaccharide donor $\mathbf{1 5}$ with the disaccharide acceptor $\mathbf{5}$ gave the pentasaccharide $\mathbf{2 0}$ in satisfactory yield $(85 \%)$. Subsequent hydrolysis to remove the ethylidene group, followed by acetylation and deacylation afforded the free pentasaccharide $\mathbf{2 2}$.

The same strategy was applied for the synthesis of higher mannose oligosaccharides. Therefore, conden-

sation of the trisaccharide donor $\mathbf{1 5}$ with the trisaccharide acceptor $\mathbf{8}$ gave the hexasaccharide 23, while condensation with the tetrasaccharide acceptor $\mathbf{1 1}$ gave the heptasaccharide 26. Meanwhile, coupling of the tetrasaccharide acceptor $\mathbf{1 1}$ with the tetrasaccharide donor 19 afforded the octasaccharide 29. Deethylidenation of $\mathbf{2 3}, \mathbf{2 6}$, and $\mathbf{2 9}$, followed by deacylation gave the free hexasaccharide $\mathbf{2 5}$, heptasaccharide 28, and octasaccharide 31, respectively. To the best of our knowledge, these are the first reported syntheses of mannoheptaose $\mathbf{2 8}$ and mannooctaose $\mathbf{3 1}$.

It was found that the size of either donor or acceptor did not have a significant influence on the yield of the coupling. Owing to the use of convenient materials and the simplicity of the procedure, this method is readily amenable to large-scale production of mannose oligosaccharides.

\section{Conclusion}

In summary, we have presented herein a very facile and practical method for the synthesis of multi branched mannose oligosaccharides of $N$-glycans. Sufficient quantities of mannose oligosaccharide samples can be obtained and this will greatly facilitate the biological studies.

\section{Experimental}

\subsection{General}

Optical rotations were determined at $25^{\circ} \mathrm{C}$ with a Perkin-Elmer Model 241-Mc automatic polarimeter. Melting points were determined with a 'Mel-Temp' apparatus. ${ }^{1} \mathrm{H}$ and ${ }^{13} \mathrm{C}$ NMR spectra were recorded with Bruker ARX 400 spectrometers $(400 \mathrm{MHz}$ for ${ }^{1} \mathrm{H}, 100 \mathrm{MHz}$ for ${ }^{13} \mathrm{C}$ ) for solutions in $\mathrm{CDCl}_{3}$ or $\mathrm{D}_{2} \mathrm{O}$ as indicated. Chemical shifts are given in $\mathrm{ppm}$ downfield from internal $\mathrm{Me}_{4} \mathrm{Si}$. Mass spectra were measured using MALTI-TOF-MS with CCA as matrix or recorded with a VG PLATFORM mass spectrometer using the ESI mode. Thin-layer chromatography (TLC) was performed on silica gel $\mathrm{HF}_{254}$ with detection by charring with $30 \%(\mathrm{v} / \mathrm{v}) \mathrm{H}_{2} \mathrm{SO}_{4}$ in $\mathrm{MeOH}$ or in some cases by a UV detector. Column chromatography was conducted by elution of a column $(16 \times 240 \mathrm{~mm}, 18 \times 300 \mathrm{~mm}, 35 \times 400 \mathrm{~mm})$ of silica gel (100-200 mesh) with EtOAc-petroleum ether $\left(60-90^{\circ} \mathrm{C}\right)$ as the eluent. Solutions were concentrated at $<60^{\circ} \mathrm{C}$ under reduced pressure. In the syntheses, compound $\mathbf{2}$ containing an ethylidene group was composed of $(R)$ - and $(S)$-isomers in a 4:1 ratio. The two isomers were separated on a silica gel column with 2:1 petroleum ether-EtOAc as the eluent. Thus, pure $(R)-2$ was used in further reactions in most cases, yielding products predominantly consisting of the $(R)$-isomer as the major compound and less than $10 \%$ of the $(S)$-form as the minor isomer. An exception was the synthesis of 26, in which $(S)$-2 was used at the beginning and thus the $(S)$-form was obtained as the predominant isomer for $\mathbf{2 6}$. The $(R)$ - and $(S)$ isomers had no difference in reactivity and thus no separation was conducted in the synthesis. However, for convenience of identification by NMR spectrometry, the predominant isomer in each synthesis was isolated in pure form. The assignments of $(R)$ - and $(S)$-forms were based on the data reported by Kochetkov. $^{7}$

\subsection{4,6- $O$-Benzylidene-1,2- $O$-ethylidene- $\beta$-D-manno- pyranose 2}

$p$-Toluenesulfonic acid monohydrate (190 mg, 1 mmol) was added to a solution of $1(2.06 \mathrm{~g}, 10$ $\mathrm{mmol})$, triethyl orthoformate $(4.2 \mathrm{~mL}, 25 \mathrm{mmol})$ and benzaldehyde $(3.0 \mathrm{~mL}, 30 \mathrm{mmol})$ in anhydrous DMF $(25 \mathrm{~mL})$. The mixture was stirred at room temperature for $12 \mathrm{~h}$, when TLC $(2: 1$ petroleum etherEtOAc) indicated that the reaction was complete. Sodium bicarbonate $(2.52 \mathrm{~g}, 30 \mathrm{mmol})$ was added to the reaction mixture, and the mixture was stirred for an additional hour. After filtration, the solvents were evaporated in vacuo to give a residue, which was subjected to silica gel column chromatography (2:1 petroleum ether-EtOAc) to give $\mathbf{2}$ as a white solid $(2.58 \mathrm{~g}, 88 \%)$ consisting of $(R)$ - and $(S)$-isomers in a $4: 1$ ratio. Separation of the mixture with $2: 1$ petroleum ether-EtOAc as the eluent gave pure $(R)$ and $(S)$-isomers. For the $(R)$-isomer: $[\alpha]_{\mathrm{D}}-47.6(c$ 1.3, $\left.\mathrm{CHCl}_{3}\right) ;{ }^{1} \mathrm{H}$ NMR $\left(400 \mathrm{MHz}, \mathrm{CDCl}_{3}\right) \delta 7.37-$ $7.50(\mathrm{~m}, 5 \mathrm{H}, P h), 5.58(\mathrm{~s}, 1 \mathrm{H}, \mathrm{PhCH}), 5.33(\mathrm{q}, 1 \mathrm{H}$, $J=4.8, \mathrm{MeC} H), 5.26\left(\mathrm{~d}, 1 \mathrm{H}, J_{1,2}=2.0, \mathrm{H}-1\right), 4.31$ $\left(\mathrm{dd}, 1 \mathrm{H}, J_{5,6}=5.1, J_{6,6^{\prime}}=10.6, \mathrm{H}-6\right), 4.20(\mathrm{dd}, 1 \mathrm{H}$, $\left.J_{1,2}=2.0, \quad J_{2,3}=3.2, \quad \mathrm{H}-2\right), 4.07 \quad\left(\mathrm{dd}, \quad 1 \mathrm{H}, \quad J_{2,3}=3.2\right.$, $\left.J_{3,4}=9.4, \mathrm{H}-3\right), 3.92\left(\mathrm{dd}, 1 \mathrm{H}, J_{3,4}=J_{4,5}=9.4, \mathrm{H}-4\right)$, $3.77\left(\mathrm{dd}, 1 \mathrm{H}, J_{5,6^{\prime}}=2.7, J_{6,6^{\prime}}=10.6, \mathrm{H}-6^{\prime}\right), 3.38$ (ddd, $\left.1 \mathrm{H}, J_{4,5}=9.4, J_{5,6}=5.1, J_{5,6^{\prime}}=2.7, \mathrm{H}-5\right), 2.44-2.48(\mathrm{br}$, $1 \mathrm{H}, \mathrm{OH}), 1.52(\mathrm{~d}, 3 \mathrm{H}, J=4.9, M e \mathrm{CH})$. Anal. calcd for $\mathrm{C}_{15} \mathrm{H}_{18} \mathrm{O}_{6}: \mathrm{C}, 61.21 ; \mathrm{H}, 6.16$. Found: $\mathrm{C}, 61.32 ; \mathrm{H}$, $6.25 \%$. For the $(S)$-isomer: $[\alpha]_{\mathrm{D}}-42.1\left(c 1.1, \mathrm{CHCl}_{3}\right)$; ${ }^{1} \mathrm{H}$ NMR (400 $\left.\mathrm{MHz}, \mathrm{CDCl}_{3}\right) \delta 7.33-7.51(\mathrm{~m}, 5 \mathrm{H}$, $P h), \quad 5.57$ (q, $1 \mathrm{H}, J=4.8, \mathrm{MeCH}), 5.56(\mathrm{~s}, 1 \mathrm{H}$, $\mathrm{PhCH}), 5.40\left(\mathrm{~d}, 1 \mathrm{H}, J_{1,2}=1.9, \mathrm{H}-1\right), 4.00(\mathrm{dd}, 1 \mathrm{H}$, $\left.J_{5,6}=5.1, J_{6,6^{\prime}}=10.6, \mathrm{H}-6\right), 3.83-3.73(\mathrm{~m}, 4 \mathrm{H}, \mathrm{H}-2, \mathrm{H}-$ $3, \mathrm{H}-4$ and $\mathrm{H}-6^{\prime}$ ), 3.35 (ddd, $1 \mathrm{H}, J_{4,5}=9.4, J_{5,6}=5.1$, $\left.J_{5,6^{\prime}}=2.7, \mathrm{H}-5\right), 2.44-2.48(\mathrm{br}, 1 \mathrm{H}, \mathrm{OH}), 1.36(\mathrm{~d}, 3 \mathrm{H}$, $J=4.9, M e \mathrm{CH}$ ). Anal. calcd for $\mathrm{C}_{15} \mathrm{H}_{18} \mathrm{O}_{6}: \mathrm{C}, 61.21$; $\mathrm{H}, 6.16$. Found: C, 61.18; H, 6.15\%. 
4.3. $2,3,4,6$-Tetra- $O$-benzoyl- $\alpha$-D-mannopyranosyl- $(1 \rightarrow$ 3)-4,6-O-benzylidene-1,2- $O$-ethylidene- $\beta$-D-mannopyranose 4

To a cooled solution $\left(0^{\circ} \mathrm{C}\right)$ of $2(2.94 \mathrm{~g}, 10 \mathrm{mmol})$ and $3(7.40 \mathrm{~g}, 10 \mathrm{mmol})$ in anhydrous $\mathrm{CH}_{2} \mathrm{Cl}_{2}(50 \mathrm{~mL})$ was added TMSOTf $(50 \mu \mathrm{L}, 0.28 \mathrm{mmol})$. The mixture was stirred at this temperature for $2 \mathrm{~h}$, and then quenched with $\mathrm{Et}_{3} \mathrm{~N}$ (two drops). The solvents were evaporated in vacuo to give a residue, which was purified by silica gel column chromatography (2:1 petroleum ether-EtOAc) to give disaccharide 4 as a syrup $(4.72 \mathrm{~g}, 83 \%)$. For the $(R)$-isomer: $[\alpha]_{\mathrm{D}}-39.7\left(c\right.$ 1.1, $\left.\mathrm{CHCl}_{3}\right) ;{ }^{1} \mathrm{H}$ NMR (400 $\left.\mathrm{MHz}, \mathrm{CDCl}_{3}\right) \delta 8.07-7.37(\mathrm{~m}, 25 \mathrm{H}, 5 P h), 6.07(\mathrm{dd}, 1 \mathrm{H}$, $\left.J_{3,4}=J_{4,5}=9.8, \mathrm{H}-4\right), 6.01\left(\mathrm{dd}, 1 \mathrm{H}, J_{2,3}=3.0, J_{3,4}=9.8\right.$, $\mathrm{H}-3), 5.86\left(\mathrm{dd}, 1 \mathrm{H}, J_{1,2}=1.0, J_{2,3}=3.0, \mathrm{H}-2\right), 5.66(\mathrm{~s}$, $1 \mathrm{H}, \mathrm{PhCH}), 5.59\left(\mathrm{~d}, 1 \mathrm{H}, J_{1,2}=1.0, \mathrm{H}-1\right), 5.41(\mathrm{q}, 1 \mathrm{H}$, $J=4.7, \mathrm{MeC} H), 5.07\left(\mathrm{~d}, 1 \mathrm{H}, J_{1,2}=1.6, \mathrm{H}-1\right), 4.69(\mathrm{dd}$, $\left.1 \mathrm{H}, J_{2,3}=2.0, J_{3,4}=9.7, \mathrm{H}-3\right), 4.62\left(\mathrm{ddd}, 1 \mathrm{H}, J_{4,5}=9.8\right.$, $\left.J_{5,6}=4.2, J_{5,6^{\prime}}=2.6, \mathrm{H}-5\right), 4.70\left(\mathrm{dd}, 1 \mathrm{H}, J_{5,6}=4.5, J_{6,6^{\prime}}=\right.$ 13.5, H-6), 4.34-4.23 (m, 4H, H-2, H-6 and 2H-6'), 3.81 $\left(\mathrm{dd}, 1 \mathrm{H}, J_{3,4}=J_{4,5}=9.7, \mathrm{H}-4\right), 3.36\left(\mathrm{ddd}, 1 \mathrm{H}, J_{4,5}=9.7\right.$, $\left.J_{5,6}=4.5, J_{5,6^{\prime}}=4.3, \mathrm{H}-5\right), 1.58(\mathrm{~d}, 3 \mathrm{H}, J=4.7, M e \mathrm{CH})$. Anal. calcd for $\mathrm{C}_{49} \mathrm{H}_{44} \mathrm{O}_{15}$ : C, 67.42; H, 5.08. Found: $\mathrm{C}$, 67.56; H, 5.12\%.

\section{4. $\quad 2,3,4,6$-Tetra- $O$-benzoyl- $\alpha$-D-mannopyranosyl-( $1 \rightarrow$ 3)-1,2-O-ethylidene- $\beta$-D-mannopyranose 5}

To a solution of $4(8.72 \mathrm{~g}, 10.0 \mathrm{mmol})$ in anhydrous $\mathrm{MeOH}(100 \mathrm{~mL})$ was added acetyl chloride $(0.1 \mathrm{~mL})$. The solution was sealed in a flask and stirred at $\mathrm{rt}$ until TLC (2:1 petroleum ether-EtOAc) showed that all starting material was consumed. The solution was neutralized with $\mathrm{Et}_{3} \mathrm{~N}$, then concentrated to dryness. The residue was passed through a short silica gel column (1:2 petroleum ether-EtOAc) to give $\mathbf{5}$ as a white solid $(6.90 \mathrm{~g}, 88 \%)$. For the $(R)$-isomer: $[\alpha]_{\mathrm{D}}-49.7$ (c 1.2, $\left.\mathrm{CHCl}_{3}\right) ;{ }^{1} \mathrm{H}$ NMR $\left(400 \mathrm{MHz}, \mathrm{CDCl}_{3}\right) \delta 8.08-7.27(\mathrm{~m}$, $20 \mathrm{H}, 4 P h), 6.13\left(\mathrm{dd}, 1 \mathrm{H}, J_{3,4}=J_{4,5}=10.2, \mathrm{H}-4\right), 5.97$ $\left(\mathrm{dd}, 1 \mathrm{H}, J_{2,3}=3.4, J_{3,4}=10.2, \mathrm{H}-3\right), 5.83\left(\mathrm{dd}, 1 \mathrm{H}, J_{1,2}=\right.$ $\left.1.1, J_{2,3}=3.4, \mathrm{H}-2\right), 5.42\left(\mathrm{~d}, 1 \mathrm{H}, J_{1,2}=1.1, \mathrm{H}-1\right), 5.31(\mathrm{q}$, $1 \mathrm{H}, J=4.8, \mathrm{MeC} H), 5.23\left(\mathrm{~d}, 1 \mathrm{H}, J_{1,2}=2.1, \mathrm{H}-1\right), 4.74$ (ddd, $1 \mathrm{H}, J_{4,5}=10.2, J_{5,6}=4.4, J_{5,6^{\prime}}=2.3, \mathrm{H}-5$ ), 4.65 $\left(\mathrm{dd}, 1 \mathrm{H}, J_{2,3}=2.4, J_{3,4}=9.4, \mathrm{H}-3\right), 4.51\left(\mathrm{dd}, 1 \mathrm{H}, J_{5,6}=\right.$ $\left.4.4, J_{6,6^{\prime}}=12.1, \mathrm{H}-6\right), 4.38\left(\mathrm{dd}, 1 \mathrm{H}, J_{1,2}=2.0, J_{2,3}=2.4\right.$, $\mathrm{H}-2) 4.17\left(\mathrm{dd}, 1 \mathrm{H}, J_{3,4}=J_{4,5}=9.4, \mathrm{H}-4\right), 3.91-3.83(\mathrm{~m}$, $3 \mathrm{H}, \mathrm{H}-6$, and $2 \mathrm{H}-6^{\prime}$ ), 3.43 (ddd, $1 \mathrm{H}, J_{4,5}=9.4, J_{5,6}=5.1$, $\left.J_{5,6^{\prime}}=2.2, \mathrm{H}-5\right), 1.53(\mathrm{~d}, 3 \mathrm{H}, J=4.8, M e \mathrm{CH}) ;{ }^{13} \mathrm{C} \mathrm{NMR}$ $\left(100 \mathrm{MHz}, \mathrm{CDCl}_{3}\right) \delta 165.7,165.3,165.1,164.9(4 \mathrm{C}$, 4PhCO), 133.0-127.9 (PhCO), $103.9\left(\mathrm{CH}_{3} \mathrm{CH}\right), 99.5$, 96.4 (2C-1), 81.3, 78.9, 74.8, 69.9, 69.8, 69.0, 66.4, 65.8, 62.5, 61.9 (10C, C-2-6), $21.3\left(\mathrm{CH}_{3} \mathrm{CH}\right)$. Anal. calcd for $\mathrm{C}_{42} \mathrm{H}_{40} \mathrm{O}_{15}: \mathrm{C}, 64.28 ; \mathrm{H}$, 5.14. Found: C, 64.24; H, $5.15 \%$.

4.5. 2- $O$-Acetyl-3,4,6-tri- $O$-benzoyl- $\alpha$-D-mannopyranosyl-( $1 \rightarrow 2)-3,4,6$-tri- $O$-benzoyl- $\alpha$-D-mannopyranosyl-(1 $\rightarrow 3$ )-4,6- $O$-benzylidene-1,2- $O$-ethylidene- $\beta$-D-mannopyranose 7

To a cooled solution $\left(0^{\circ} \mathrm{C}\right)$ of $2(0.74 \mathrm{~g}, 2.5 \mathrm{mmol})$ and $6(2.88 \mathrm{~g}, 2.5 \mathrm{mmol})$ in anhydrous $\mathrm{CH}_{2} \mathrm{Cl}_{2}(50 \mathrm{~mL})$ was added TMSOTf $(25 \mu \mathrm{L}, 0.14 \mathrm{mmol})$. The mixture was stirred at this temperature for $2 \mathrm{~h}$, and then quenched with $\mathrm{Et}_{3} \mathrm{~N}$ (two drops). After evaporation of the solvents, the crude residue was subjected to silica gel column chromatography (2:1 petroleum ether-EtOAc) to give trisaccharide 7 as a foamy solid $(2.94 \mathrm{~g}, 92 \%)$. For the $(R)$-isomer: $[\alpha]_{\mathrm{D}}^{25}-31.9\left(c \quad 1.0, \mathrm{CHCl}_{3}\right) ;{ }^{1} \mathrm{H}$ NMR $\left(400 \mathrm{MHz}, \mathrm{CDCl}_{3}\right) \delta 8.06-7.18(\mathrm{~m}, 35 \mathrm{H}, 7 \mathrm{Ph})$, $5.98\left(\mathrm{dd}, 1 \mathrm{H}, J_{2,3}=3.1, J_{3,4}=9.6, \mathrm{H}-3\right), 5.95(\mathrm{dd}, 1 \mathrm{H}$, $\left.J_{3,4}=J_{3,4}=9.6, \mathrm{H}-4\right), 5.89\left(\mathrm{dd}, 1 \mathrm{H}, J_{2,3}=3.2, J_{3,4}=9.8\right.$, $\mathrm{H}-3), 5.78\left(\mathrm{dd}, 1 \mathrm{H}, J_{3,4}=J_{3,4}=9.8, \mathrm{H}-4\right), 5.66(\mathrm{dd}, 1 \mathrm{H}$, $\left.J_{1,2}=1.6, J_{2,3}=3.1, \mathrm{H}-2\right), 5.58\left(\mathrm{~d}, 1 \mathrm{H}, J_{1,2}=1.6, \mathrm{H}-1\right)$, $5.37(\mathrm{q}, 1 \mathrm{H}, J=4.9, \mathrm{MeCH}), 5.34(\mathrm{~s}, 1 \mathrm{H}, \mathrm{PhCH}), 5.06$ $\left(\mathrm{d}, 1 \mathrm{H}, J_{1,2}=1.4, \mathrm{H}-1\right), 4.93\left(\mathrm{~d}, 1 \mathrm{H}, J_{1,2}=0.9, \mathrm{H}-1\right), 4.68$ $\left(\mathrm{dd}, 1 \mathrm{H}, J_{2,3}=3.2, J_{3,4}=10.0, \mathrm{H}-3\right), 4.63\left(\mathrm{dd}, 1 \mathrm{H}, J_{5,6}=\right.$ $\left.4.9, J_{6,6^{\prime}}=11.7, \mathrm{H}-6\right), 4.54\left(\mathrm{ddd}, 1 \mathrm{H}, J_{4,5}=9.4, J_{5,6}=5.1\right.$, $\left.J_{5,6^{\prime}}=3.3, \mathrm{H}-5\right), 4.48\left(\mathrm{dd}, 1 \mathrm{H}, J_{1,2}=1.9, J_{2,3}=3.1, \mathrm{H}-2\right)$, $4.31\left(\mathrm{ddd}, 1 \mathrm{H}, J_{4,5}=9.6, J_{5,6}=4.9, J_{5,6^{\prime}}=4.3, \mathrm{H}-5\right), 4.19$ $\left(\mathrm{dd}, 1 \mathrm{H}, J_{5,6}=5.1, J_{6,6^{\prime}}=10.6, \mathrm{H}-6\right), 4.13-4.00(\mathrm{~m}, 5 \mathrm{H})$, $3.65\left(\mathrm{dd}, 1 \mathrm{H}, J_{3,4}=J_{3,4}=10.0, \mathrm{H}-4\right), 3.13(\mathrm{ddd}, 1 \mathrm{H}$, $\left.J_{4,5}=9.8, \quad J_{5,6}=5.5, \quad J_{5,6^{\prime}}=2.4, \quad \mathrm{H}-5\right), 2.03 \quad(\mathrm{~s}, \quad 3 \mathrm{H}$, $\left.\mathrm{CH}_{3} \mathrm{CO}\right), 1.56(\mathrm{~d}, 1 \mathrm{H}, J=4.9, \mathrm{MeCH}) ;{ }^{13} \mathrm{C}$ NMR $(100$ $\left.\mathrm{MHz}, \mathrm{CDCl}_{3}\right) \delta 168.8\left(\mathrm{CH}_{3} \mathrm{CO}\right), 165.8,165.3,165.2$, 165.1, 165.0, 164.6 (6C, PhCO), 136.5-125.5 ( $\mathrm{PhCO}$ and $\mathrm{PhCH}), 104.3\left(\mathrm{CH}_{3} \mathrm{CH}\right), 101.1(\mathrm{PhCH}), 99.5,98.6$, 96.4 (3C-1), 79.2, 75.7, 73.6, 70.1, 69.2, 69.2, 68.8, 68.1, 67.7, 66.6, 65.1, 63.6, 62.5 (15C, C-2-6, some signals overlapped), 21.3, $20.1\left(\mathrm{CH}_{3} \mathrm{CH}\right.$ and $\left.\mathrm{CH}_{3} \mathrm{CO}\right)$. Anal. calcd for $\mathrm{C}_{71} \mathrm{H}_{64} \mathrm{O}_{23}: \mathrm{C}, 66.35 ; \mathrm{H}, 5.02$. Found: $\mathrm{C}$, 66.48 ; $\mathrm{H}, 4.93 \%$.

4.6. 2- $O$-Acetyl-3,4,6-tri- $O$-benzoyl- $\alpha$-D-mannopyranosyl-( $1 \rightarrow 2)-3,4,6$-tri- $O$-benzoyl- $\alpha$-D-mannopyranosyl-(1 $\rightarrow 3$ )-1,2- $O$-ethylidene- $\beta$-D-mannopyranose 8

To a solution of $7(2.57 \mathrm{~g}, 2 \mathrm{mmol})$ in anhydrous $\mathrm{MeOH}(100 \mathrm{~mL})$ was added acetyl chloride $(0.1 \mathrm{~mL})$. The solution was sealed in a flask and stirred at room temperature until TLC (2:1 petroleum ether-EtOAc) showed that the starting material disappeared. The solution was neutralized with $\mathrm{Et}_{3} \mathrm{~N}$, then concentrated to dryness. The residue was passed through a short silica gel column (1:2 petroleum ether-EtOAc) to give 8 as a white solid $(2.27 \mathrm{~g}, 95 \%)$. For the $(R)$-isomer: $[\alpha]_{\mathrm{D}}^{25}$ $-3.8\left(c \quad 0.5, \mathrm{CHCl}_{3}\right) ;{ }^{1} \mathrm{H}$ NMR $\left(400 \mathrm{MHz}, \mathrm{CDCl}_{3}\right) \delta$ 8.06-7.18 (m, 30H, 6Ph), 6.05-5.86 (m, 4H, 2H-3, $2 \mathrm{H}-4), 5.63\left(\mathrm{dd}, 1 \mathrm{H}, J_{1,2}=1.5, J_{2,3}=3.1, \mathrm{H}-2\right), 5.58(\mathrm{~d}$, $\left.1 \mathrm{H}, J_{1,2}=1.5, \mathrm{H}-1\right), 5.24(\mathrm{q}, 1 \mathrm{H}, J=4.7, \mathrm{MeC} H), 5.16$ $\left(\mathrm{d}, 1 \mathrm{H}, J_{1,2}=1.7, \mathrm{H}-1\right), 5.04\left(\mathrm{~d}, 1 \mathrm{H}, J_{1,2}=2.2, \mathrm{H}-1\right)$, $4.644 .69(\mathrm{~m}, 7 \mathrm{H}), 4.18\left(\mathrm{dd}, 1 \mathrm{H}, J_{1,2}=2.2, J_{2,3}=3.6\right.$, $\mathrm{H}-2), 4.08\left(\mathrm{dd}, 1 \mathrm{H}, J_{3,4}=J_{3,4}=9.5, \mathrm{H}-4\right), 3.83-3.76(\mathrm{~m}$, $3 \mathrm{H}), 3.26\left(\mathrm{ddd}, 1 \mathrm{H}, J_{4,5}=9.5, J_{5,6}=5.1, J_{5,6^{\prime}}=2.3, \mathrm{H}-5\right.$ ), 2.04 (s, 3H, $\left.\mathrm{CH}_{3} \mathrm{CO}\right), 1.49(\mathrm{~d}, 1 \mathrm{H}, J=4.7, \mathrm{MeCH}) ;{ }^{13} \mathrm{C}$ NMR $\left(100 \mathrm{MHz}, \mathrm{CDCl}_{3}\right) \delta 168.9\left(\mathrm{CH}_{3} \mathrm{CO}\right), 166.0$, $165.8,165.4,165.2,164.9,164.7$ (6C, PhCO), 133.0127.9 (PhCO), $103.9\left(\mathrm{CH}_{3} \mathrm{CH}\right), 100.3,98.7,96.3(3 \mathrm{C}$, 3C-1), 79.6, 78.8, 74.8, 70.4, 69.2, 68.9, 67.2, 67.0, 66.1, 63.4, 63.0, 61.8 (15C, 3C-2-6, some signals overlapped), $21.2\left(\mathrm{CH}_{3} \mathrm{CH}\right), 20.1\left(\mathrm{CH}_{3} \mathrm{CO}\right)$. Anal. calcd for $\mathrm{C}_{42} \mathrm{H}_{40} \mathrm{O}_{15}$ : C, 64.21; H, 5.05. Found: C, 64.04; H, $4.90 \%$. 
4.7. $\quad 2,3,4,6$-Tetra- $O$-benzoyl- $\alpha$-D-mannopyranosyl- $(1 \rightarrow$ 2)-3,4,6-tri- $O$-benzoyl- $\alpha$-D-mannopyranosyl- $(1 \rightarrow 2)-3,4,6$ tri- $O$-benzoyl- $\alpha$-D-mannopyranosyl- $(1 \rightarrow 3)-4,6-O$ benzylidene-1,2- $O$-ethylidene- $\beta$-D-mannopyranose 10

TMSOTf $(25 \mu \mathrm{L}, 0.14 \mathrm{mmol})$ was added to a cooled solution $\left(0^{\circ} \mathrm{C}\right)$ of $2(0.59 \mathrm{~g}, 2.0 \mathrm{mmol})$ and $9(3.38 \mathrm{~g}, 2.0$ $\mathrm{mmol})$ in anhydrous $\mathrm{CH}_{2} \mathrm{Cl}_{2}(50 \mathrm{~mL})$. The mixture was stirred at this temperature for $2 \mathrm{~h}$, and then quenched with $\mathrm{Et}_{3} \mathrm{~N}$ (two drops). The solvents were evaporated in vacuo to give a residue, which was purified by silica gel column chromatography (2:1 petroleum ether-EtOAc) to give tetrasaccharide $\mathbf{1 0}$ as a syrup $(3.17 \mathrm{~g}, 86 \%)$. For the $(R)$-isomer: $[\alpha]_{\mathrm{D}}^{25}-33.1\left(c \quad 1.2, \mathrm{CHCl}_{3}\right) ;{ }^{1} \mathrm{H}$ NMR $\left(400 \mathrm{MHz}, \mathrm{CDCl}_{3}\right) \delta 8.11-7.27(\mathrm{~m}, 55 \mathrm{H}, 11 \mathrm{Ph}), 6.06$ $\left(\mathrm{dd}, 1 \mathrm{H}, J_{3,4}=J_{4.5}=9.6, \mathrm{H}-4\right), 5.99-5.94(\mathrm{~m}, 2 \mathrm{H}, 2 \mathrm{H}-3)$, $5.93\left(\mathrm{dd}, 1 \mathrm{H}, J_{3.4}=J_{3.4}=9.8, \mathrm{H}-4\right), 5.88\left(\mathrm{dd}, 1 \mathrm{H}, J_{2,3}=\right.$ $\left.3.0, J_{3,4}=9.8, \mathrm{H}-3\right), 5.81\left(\mathrm{dd}, 1 \mathrm{H}, J_{1,2}=1.5, J_{2,3}=3.1\right.$, $\mathrm{H}-2), 5.73\left(\mathrm{dd}, 1 \mathrm{H}, J_{3,4}=J_{3,4}=9.7, \mathrm{H}-4\right), 5.60(\mathrm{~d}, 1 \mathrm{H}$, $\left.J_{1,2}=1.6, \mathrm{H}-1\right), 5.52(\mathrm{~s}, 1 \mathrm{H}, \mathrm{PhCH}), 5.38\left(\mathrm{~d}, 1 \mathrm{H}, J_{1,2}=\right.$ $1.0, \mathrm{H}-1), 5.31(\mathrm{q}, 1 \mathrm{H}, J=4.7, \mathrm{MeC} H), 5.11(\mathrm{~d}, 1 \mathrm{H}$, $\left.J_{1,2}=1.5, \mathrm{H}-1\right), 4.99\left(\mathrm{~d}, 1 \mathrm{H}, J_{1,2}=1.9, \mathrm{H}-1\right), 4.74(\mathrm{dd}$, $\left.1 \mathrm{H}, J_{2,3}=3.2, J_{3,4}=9.7, \mathrm{H}-3\right), 4.65\left(\mathrm{dd}, 1 \mathrm{H}, J_{5,6}=5.2\right.$, $\left.J_{6,6^{\prime}}=11.8, \mathrm{H}-6\right), 4.54\left(\mathrm{ddd}, 1 \mathrm{H}, J_{4,5}=9.4, J_{5,6}=5.0\right.$, $\left.J_{5,6^{\prime}}=2.3, \mathrm{H}-5\right), 4.50-4.45(\mathrm{~m}, 3 \mathrm{H}), 4.28$ (ddd, $1 \mathrm{H}$, $\left.J_{4,5}=9.6, J_{5,6}=5.0, J_{5,6^{\prime}}=2.1, \mathrm{H}-5\right), 4.20\left(\mathrm{dd}, 1 \mathrm{H}, J_{5,6}=\right.$ 4.9, $\left.J_{6,6^{\prime}}=10.5, \mathrm{H}-6\right), 4.15-4.04(\mathrm{~m}, 7 \mathrm{H}), 3.82(\mathrm{dd}, 1 \mathrm{H}$, $\left.J_{3,4}=J_{3,4}=9.9, \mathrm{H}-4\right), 3.40\left(\mathrm{ddd}, 1 \mathrm{H}, J_{4,5}=9.8, J_{5,6}=5.4\right.$, $\left.J_{5,6^{\prime}}=2.6, \mathrm{H}-5\right), 1.52(\mathrm{~d}, 1 \mathrm{H}, J=4.9, M e \mathrm{CH}) ;{ }^{13} \mathrm{C} \mathrm{NMR}$ $\left(100 \mathrm{MHz}, \mathrm{CDCl}_{3}\right) \delta 165.9,165.8,165.4,165.3,165.2$, $165.1,165.0,164.4(10 \mathrm{C}, 10 \mathrm{PhCO}$, some signals overlapped), 136.5-125.5 (PhCO and $P h \mathrm{CH}), 103.9$ $\left(\mathrm{CH}_{3} \mathrm{CH}\right), 100.8(\mathrm{PhCH}), 99.1,99.0,97.3,96.9(4 \mathrm{C}$, 4C-1), 79.8, 75.5, 74.9, 73.5, 73.6, 70.1, 69.3, 69.2, 68.9, $68.1,67.0,66.7,65.4,64.0,62.1$ (20C, 4C-2-6, some signals overlapped), $21.3\left(\mathrm{CH}_{3} \mathrm{CH}\right)$. Anal. calcd for $\mathrm{C}_{103} \mathrm{H}_{88} \mathrm{O}_{31}: \mathrm{C}, 67.91 ; \mathrm{H}, 4.87$. Found: $\mathrm{C}, 68.12 ; \mathrm{H}$, 4.85 .

\subsection{2,3,4,6-Tetra- $O$-benzoyl- $\alpha$-D-mannopyranosyl-( $1 \rightarrow$ 2)-3,4,6-tri- $O$-benzoyl- $\alpha$-D-mannopyranosyl-( $1 \rightarrow 2)-3,4,6$ - tri- $O$-benzoyl- $\alpha$-D-mannopyranosyl-( $1 \rightarrow 3)-1,2-O$-ethyl- idene- $\beta$-D-mannopyranose 11}

To a solution of $\mathbf{1 0}(3.00 \mathrm{~g}, 1.65 \mathrm{mmol})$ in anhydrous $\mathrm{MeOH}(100 \mathrm{~mL})$ was added acetyl chloride $(0.1 \mathrm{~mL})$. The solution was sealed in a flask and stirred at $\mathrm{rt}$ until TLC (1:1 petroleum ether-EtOAc) showed that the starting material disappeared. The solution was neutralized with $\mathrm{Et}_{3} \mathrm{~N}$, and then concentrated. Purification of the residue on a short silica gel column (1:2 petroleum ether-EtOAc) gave acceptor $\mathbf{1 1}$ as a white foam $(2.60 \mathrm{~g}$, $92 \%)$. For the $(R)$-isomer: $[\alpha]_{\mathrm{D}}^{25}-33.3\left(c 0.5, \mathrm{CHCl}_{3}\right)$; ${ }^{1} \mathrm{H}$ NMR $\left(400 \mathrm{MHz}, \mathrm{CDCl}_{3}\right) \delta$ 8.06-7.18 $(\mathrm{m}, 50 \mathrm{H}$, $10 \mathrm{Ph}), 6.10\left(\mathrm{dd}, 1 \mathrm{H}, J_{3,4}=J_{4.5}=10.1, \mathrm{H}-4\right), 5.99(\mathrm{dd}$, $\left.1 \mathrm{H}, J_{3,4}=J_{4,5}=9.9, \mathrm{H}-4\right), 5.95-5.93(\mathrm{~m}, 2 \mathrm{H}, \mathrm{H}-2$ and $\mathrm{H}-3), 5.84-5.80(\mathrm{~m}, 3 \mathrm{H}, 2 \mathrm{H}-3$ and $\mathrm{H}-4), 5.57(\mathrm{~d}, 1 \mathrm{H}$, $\left.J_{1,2}=2.1, \mathrm{H}-1\right), 5.53\left(\mathrm{~d}, 1 \mathrm{H}, J_{1,2}=1.0, \mathrm{H}-1\right), 5.23(\mathrm{q}, 1 \mathrm{H}$, $J=4.8, \mathrm{MeCH}), 5.05$ (2d, 2H, 2H-1, overlapped), 4.63$4.50(\mathrm{~m}, 9 \mathrm{H}), 4.39\left(\mathrm{dd}, 1 \mathrm{H}, J_{1,2}=1.0, J_{2.3}=3.1, \mathrm{H}-2\right)$, $4.24\left(\mathrm{dd}, 1 \mathrm{H}, J_{2,3}=3.1, J_{3,4}=9.6, \mathrm{H}-3\right), 4.17(\mathrm{dd}, 1 \mathrm{H}$, $\left.J_{1,2}=2.1, J_{2,3}=3.1, \mathrm{H}-2\right), 4.11\left(\mathrm{dd}, 1 \mathrm{H}, J_{3,4}=J_{3,4}=9.7\right.$, $\mathrm{H}-4), 3.89-3.78(\mathrm{~m}, 3 \mathrm{H}), 3.26\left(\mathrm{ddd}, 1 \mathrm{H}, J_{4,5}=9.6, J_{5,6}=\right.$ $\left.5.3, J_{5,6^{\prime}}=2.0, \mathrm{H}-5\right), 1.45(\mathrm{~d}, 1 \mathrm{H}, J=4.8, M e \mathrm{CH}) ;{ }^{13} \mathrm{C}$ NMR (100 MHz, $\left.\mathrm{CDCl}_{3}\right) \delta 166.2,165.9,165.7,165.3$, 165.2, 165.1, 165.0, 164.9, 164.6, 164.4 (10C, PhCO), 132.9-127.8 (PhCO), 103.8 $\left(\mathrm{CH}_{3} \mathrm{CH}\right), 100.2,99.1,98.6$, 96.3 (4C, 4C-1), 78.9, 78.8, 75.0, 70.0, 69.8, 69.6, 69.4, $69.1,69.0,68.9,67.8,67.4,66.8,66.2,63.5,63.3,62.2$, 62.0 (20C, 4C-2-6, some signals overlapped), 21.1 $\left(\mathrm{CH}_{3} \mathrm{CH}\right)$. Anal. calcd for $\mathrm{C}_{42} \mathrm{H}_{40} \mathrm{O}_{15}: \mathrm{C}, 66.51 ; \mathrm{H}, 4.88$. Found: C, 66.32; H, 4.96\%.

\subsection{2,3,4,6-Tetra- $O$-benzoyl- $\alpha$-D-mannopyranosyl-( $1 \rightarrow$ $3)$-[2,3,4,6-tetra- $O$-benzoyl- $\alpha$-D-mannopyranosyl-( $1 \rightarrow 6)]-$ 1,2- $O$-ethylidene- $\beta$-D-mannopyranose 12}

To a cooled solution $\left(0^{\circ} \mathrm{C}\right)$ of $5(3.92 \mathrm{~g}, 5 \mathrm{mmol})$ and $\mathbf{3}$ $(3.70 \mathrm{~g}, 5 \mathrm{mmol})$ in anhydrous $\mathrm{CH}_{2} \mathrm{Cl}_{2}(50 \mathrm{~mL})$ was added TMSOTf $(50 \mu \mathrm{L}, 0.28 \mathrm{mmol})$. The mixture was stirred at this temperature for $2 \mathrm{~h}$, and then quenched with $\mathrm{Et}_{3} \mathrm{~N}$ (four drops). The solvents were evaporated in vacuo to give a residue, which was purified by silica gel column chromatography (2:1 petroleum etherEtOAc) to give trisaccharide $\mathbf{1 2}$ as a syrup (5.92 g, $87 \%)$. For the $(R)$-isomer: $[\alpha]_{\mathrm{D}}^{25}-42.2\left(c 1.0, \mathrm{CHCl}_{3}\right)$; ${ }^{1} \mathrm{H}$ NMR $\left(400 \mathrm{MHz}, \mathrm{CDCl}_{3}\right) \delta 8.06-7.28(\mathrm{~m}, 40 \mathrm{H}$, $8 \mathrm{Ph}$ ), $6.18\left(\mathrm{dd}, 1 \mathrm{H}, J_{3,4}=J_{3,4}=9.8, \mathrm{H}-4\right), 6.15(\mathrm{dd}, 1 \mathrm{H}$, $\left.J_{3,4}=J_{3,4}=9.6, \mathrm{H}-4\right), 6.03\left(\mathrm{dd}, 1 \mathrm{H}, J_{2,3}=2.9, J_{3.4}=9.8\right.$, $\mathrm{H}-3), 5.98\left(\mathrm{dd}, 1 \mathrm{H}, J_{2,3}=3.1, J_{3,4}=9.6, \mathrm{H}-3\right), 5.89(\mathrm{dd}$, $\left.1 \mathrm{H}, J_{1,2}=0.9, J_{2,3}=3.0, \mathrm{H}-2\right), 5.81\left(\mathrm{dd}, 1 \mathrm{H}, J_{1,2}=1.2\right.$, $\left.J_{2,3}=3.1, \mathrm{H}-2\right), 5.44\left(\mathrm{~d}, 1 \mathrm{H}, J_{1,2}=0.9, \mathrm{H}-1\right), 5.37(\mathrm{q}, 1 \mathrm{H}$, $J=4.7, \mathrm{MeCH}), 5.28\left(\mathrm{~d}, 1 \mathrm{H}, J_{1.2}=1.2, \mathrm{H}-1\right), 5.21(\mathrm{~d}$, $\left.1 \mathrm{H}, J_{1,2}=1.5, \mathrm{H}-1\right), 4.85-4.72(\mathrm{~m}, 2 \mathrm{H}), 4.67(\mathrm{dd}, 1 \mathrm{H}$, $\left.J_{2,3}=2.7, J_{3,4}=9.9, \mathrm{H}-3\right), 4.55-4.49(\mathrm{~m}, 3 \mathrm{H}), 4.45(\mathrm{dd}$, $\left.1 \mathrm{H}, J_{1,2}=1.5, J_{2,3}=2.7, \mathrm{H}-2\right), 4.24\left(\mathrm{dd}, 1 \mathrm{H}, J_{3,4}=J_{3,4}=\right.$ 9.9, H-4), 4.15 (dd, $\left.1 \mathrm{H}, J_{5,6}=4.9, J_{6,6^{\prime}}=10.8, \mathrm{H}-6\right), 3.59$ $\left(\mathrm{ddd}, 1 \mathrm{H}, J_{4,5}=9.8, J_{5,6}=4.9, J_{5,6^{\prime}}=2.2, \mathrm{H}-5\right), 1.58(\mathrm{~d}$, $1 \mathrm{H}, J=4.7, \mathrm{MeCH}) ;{ }^{13} \mathrm{C}$ NMR $\left(100 \mathrm{MHz}, \mathrm{CDCl}_{3}\right) \delta$ $165.8,165.7,165.3,165.2,165.1,165.0,164.9,164.8$ (8C, $8 \mathrm{PhCO}), 133.1-127.8(\mathrm{PhCO}$ and $\mathrm{PhCH}), 104.2$ $\left(\mathrm{CH}_{3} \mathrm{CH}\right), 99.6,97.5,96.4$ (3C, 3C-1), 82.9, 78.8, 73.7, $70.0,69.1,68.3,66.7,66.6,66.4,65.5,62.5(15 \mathrm{C}, 3 \mathrm{C}-2-$ 6 , some signals overlapped), $21.5\left(\mathrm{CH}_{3} \mathrm{CH}\right)$. Anal. calcd for $\mathrm{C}_{76} \mathrm{H}_{66} \mathrm{O}_{24}: \mathrm{C}, 66.95 ; \mathrm{H}, 4.88$. Found: $\mathrm{C}, 66.76 ; \mathrm{H}$, $4.85 \%$.

4.10. $2,3,4,6$-Tetra- $O$-benzoyl- $\alpha$-D-mannopyranosyl- $(1 \rightarrow$ $3)$-[2,3,4,6-tetra- $O$-benzoyl- $\alpha$-D-mannopyranosyl- $(1 \rightarrow 6)]-$ 2,4-di- $O$-acetyl- $\alpha$-D-mannopyranosyl trichloroacetimidate 15

Compound 12 (5.45 g, $6.69 \mathrm{mmol}$ ) was dissolved in $90 \%$ TFA $(70 \mathrm{~mL})$ and stirred for $2 \mathrm{~h}$, at the end of which time the reaction mixture was poured directly into 250 $\mathrm{mL}$ toluene and concentrated. Drying the residue under high vacuum gave a white foamy solid. This foamy solid was dissolved in pyridine $(20 \mathrm{~mL})$, and then $\mathrm{Ac}_{2} \mathrm{O}$ $(10 \mathrm{~mL})$ was added. The reaction mixture was stirred at rt for $12 \mathrm{~h}$, and TLC (4:1 petroleum ether-EtOAc) indicated that the reaction was complete. The reaction mixture was concentrated to dryness. The resultant crude product 13 was dissolved in a $1 \mathrm{M}$ solution of ammonia-methanol $(200 \mathrm{~mL})$ and stirred at $\mathrm{rt}$ for $3 \mathrm{~h}$, at the end of which time TLC (3:1 petroleum etherEtOAc) indicated that the reaction was complete. The 
solution was concentrated to give compound $\mathbf{1 4}$ as a syrup. A mixture of $\mathbf{1 4}$, trichloroacetonitrile $(4.2 \mathrm{~mL}$, $20 \mathrm{mmol}$ ), and 1,8-diazabicyclo[5.4.0]undecene (DBU) $(0.50 \mathrm{~mL}, 4.04 \mathrm{mmol})$ in dry dichloromethane $(50 \mathrm{~mL})$ was stirred under nitrogen for $3 \mathrm{~h}$ and then concentrated. The residue was purified by flash chromatography (4:1 petroleum ether-EtOAc) to give $\mathbf{1 5}$ as a white foam $\left(5.10 \mathrm{~g}, 82 \%\right.$ for four steps): $[\alpha]_{\mathrm{D}}-33.9$ (c 1.4 , $\left.\mathrm{CHCl}_{3}\right) ;{ }^{1} \mathrm{H}$ NMR $\left(400 \mathrm{MHz}, \mathrm{CDCl}_{3}\right) \delta 8.96(\mathrm{~s}, 1 \mathrm{H}$, $\left.\mathrm{CN} \mathrm{HCCl}_{3}\right), 8.06-7.27(\mathrm{~m}, 40 \mathrm{H}, 8 \mathrm{Ph}), 6.35(\mathrm{~d}, 1 \mathrm{H}$, $\left.J_{1,2}=1.4, \mathrm{H}-1\right), 6.22\left(\mathrm{dd}, 1 \mathrm{H}, J_{3,4}=J_{4,5}=9.8, \mathrm{H}-4\right), 6.11$ $\left(\mathrm{dd}, 1 \mathrm{H}, J_{3,4}=J_{4,5}=9.9, \mathrm{H}-4\right), 5.89\left(\mathrm{dd}, 1 \mathrm{H}, J_{2,3}=3.2\right.$, $\left.J_{3,4}=9.8, \mathrm{H}-3\right), 5.81\left(\mathrm{dd}, 1 \mathrm{H}, J_{2,3}=3.2, J_{3,4}=9.8, \mathrm{H}-3\right)$, $5.69\left(\mathrm{dd}, 1 \mathrm{H}, J_{1,2}=1.8, J_{2,3}=3.2, \mathrm{H}-2\right), 5.64(\mathrm{dd}, 1 \mathrm{H}$, $\left.J_{1,2}=1.5, J_{2,3}=3.1, \mathrm{H}-2\right), 5.54\left(\mathrm{dd}, 1 \mathrm{H}, J_{1,2}=1.0, J_{2,3}=\right.$ $3.1, \mathrm{H}-2), 5.51\left(\mathrm{dd}, 1 \mathrm{H}, J_{3,4}=J_{4,5}=9.8, \mathrm{H}-4\right), 5.42(\mathrm{~d}$, $\left.1 \mathrm{H}, J_{1,2}=1.8, \mathrm{H}-1\right), 5.11\left(\mathrm{~d}, 1 \mathrm{H}, J_{1,2}=1.5, \mathrm{H}-1\right), 4.68$ $\left(\mathrm{dd}, 1 \mathrm{H}, J_{2,3}=3.1, J_{3,4}=9.9, \mathrm{H}-3\right), 4.64-4.46(\mathrm{~m}, 3 \mathrm{H}$, H-5, H-6, H-6'), 4.51-4.46 (m, 3H, H-5, H-6, H-6'), $4.22\left(\mathrm{ddd}, 1 \mathrm{H}, J_{4,5}=9.8, J_{5,6}=4.7, J_{5,6^{\prime}}=2.2, \mathrm{H}-5\right), 3.99$ $\left(\mathrm{dd}, 1 \mathrm{H}, J_{5,6}=4.7, J_{6,6^{\prime}}=11.0, \mathrm{H}-6\right), 3.75\left(\mathrm{dd}, 1 \mathrm{H}, J_{5,6^{\prime}}=\right.$ 2.1, $\left.J_{6,6^{\prime}}=11.0, \mathrm{H}-6\right), 2.37$ (s, 3H, MeCO), $2.29(\mathrm{~s}, 3 \mathrm{H}$, $\mathrm{MeCO}$ ). Anal. calcd for $\mathrm{C}_{80} \mathrm{H}_{68} \mathrm{Cl}_{3} \mathrm{NO}_{26}: \mathrm{C}, 61.36 ; \mathrm{H}$, 4.38. Found: C, $61.52 ; \mathrm{H}, 4.40 \%$.

\subsection{1. $2,3,4,6$-Tetra- $O$-benzoyl- $\alpha$-D-mannopyranosyl-( $1 \rightarrow$ 3)-[2- $O$-acetyl-3,4,6-tri- $O$-benzoyl- $\alpha$-D-mannopyranosyl- $(1 \rightarrow 2)-3,4,6$-tri- $O$-benzoyl- $\alpha$-D-mannopyranosyl-( $1 \rightarrow 6)$ ]- 1,2-O-ethylidene- $\beta$-D-mannopyranose 16}

TMSOTf $(25 \mu \mathrm{L}, 0.14 \mathrm{mmol})$ was added to a cooled solution $\left(0^{\circ} \mathrm{C}\right)$ of $5(1.57 \mathrm{~g}, 2 \mathrm{mmol})$ and $\mathbf{6}(2.30 \mathrm{~g}, 2$ mmol) in anhydrous $\mathrm{CH}_{2} \mathrm{Cl}_{2}(50 \mathrm{~mL})$, and the mixture was stirred at this temperature for $2 \mathrm{~h}$ and then quenched with $\mathrm{Et}_{3} \mathrm{~N}$ (two drops). The solvents were evaporated in vacuo to give a residue, which was purified by silica gel column chromatography $(2: 1$ petroleum ether-EtOAc) to give tetrasaccharide $\mathbf{1 6}$ as a white foamy solid $(2.93 \mathrm{~g}, 83 \%)$. For the $(R)$-isomer: $[\alpha]_{\mathrm{D}}^{25}-21.6\left(c 1.3, \mathrm{CHCl}_{3}\right) ;{ }^{1} \mathrm{H}$ NMR $\left(400 \mathrm{MHz}, \mathrm{CDCl}_{3}\right)$ $\delta 8.09-7.27(\mathrm{~m}, 50 \mathrm{H}, 10 \mathrm{Ph}), 6.17\left(\mathrm{dd}, 1 \mathrm{H}, J_{3,4}=J_{4.5}=\right.$ $10.1, \mathrm{H}-4), 6.02\left(\mathrm{dd}, 1 \mathrm{H}, J_{3,4}=J_{4,5}=9.8, \mathrm{H}-4\right), 5.99(\mathrm{dd}$, $\left.1 \mathrm{H}, J_{2,3}=3.2, J_{3,4}=9.8, \mathrm{H}-3\right), 5.91-5.88(\mathrm{~m}, 4 \mathrm{H}, \mathrm{H}-2$, $2 \mathrm{H}-3$ and $\mathrm{H}-4), 5.68\left(\mathrm{dd}, 1 \mathrm{H}, J_{1,2}=1.0, J_{2,3}=2.6, \mathrm{H}-2\right)$, $5.41\left(\mathrm{~d}, 1 \mathrm{H}, J_{1,2}=1.3, \mathrm{H}-1\right), 5.37(\mathrm{q}, 1 \mathrm{H}, J=4.9$, $\mathrm{MeCH}), 5.24$ (2d, 2H, 2H-1, overlapped), 5.09 (d, 1H, $\left.J_{1,2}=1.9, \mathrm{H}-1\right), 4.45-4.68(\mathrm{~m}, 10 \mathrm{H}), 4.41\left(\mathrm{dd}, 1 \mathrm{H}, J_{1,2}=\right.$ $\left.1.3, J_{2,3}=3.1, \mathrm{H}-2\right), 4.11\left(\mathrm{dd}, 1 \mathrm{H}, J_{3,4}=J_{3,4}=9.6, \mathrm{H}-4\right)$, $4.01\left(\mathrm{dd}, 1 \mathrm{H}, J_{5,6}=5.5, J_{6,6}=10.9, \mathrm{H}-6\right), 3.90(\mathrm{dd}, 1 \mathrm{H}$, $\left.J_{2,3}=3.1, J_{3,4}=9.6, \mathrm{H}-3\right), 3.76\left(\mathrm{dd}, 1 \mathrm{H}, J_{5,6^{\prime}}=2.1, J_{6,6^{\prime}}=\right.$ $\left.10.9, \mathrm{H}-6^{\prime}\right), 3.48\left(\mathrm{ddd}, 1 \mathrm{H}, J_{4,5}=9.6, J_{5,6}=5.5, J_{5,6^{\prime}}=\right.$ 2.1, H-5), 2.02 (s, 3H, MeCO), $1.53(\mathrm{~d}, 1 \mathrm{H}, J=4.8$, $\mathrm{MeCH}) ;{ }^{13} \mathrm{C} \quad \mathrm{NMR}\left(100 \mathrm{MHz}, \mathrm{CDCl}_{3}\right) \quad \delta \quad 168.9$ (MeCO), 166.1, 165.7, 165.7, 165.3, 165.2, 165.2, 165.1, 165.0, 164.9, 164.6 (10C, PhCO), 132.1-127.8 (PhCO), $104.2\left(\mathrm{CH}_{3} \mathrm{CH}\right), 99.7,99.3,98.1,96.4(4 \mathrm{C}, 4 \mathrm{C}-1), 81.8$, $78.8,73.4,70.5,69.9,69.8,69.3,69.2,69.1,68.1,67.1$, $66.7,66.6,66.3,65.6,63.3,62.9,62.4$ (20C, 4C-2-6, some signals overlapped), $21.5 \quad(\mathrm{MeCO}), 20.1$ $\left(\mathrm{CH}_{3} \mathrm{CH}\right)$. Anal. calcd for $\mathrm{C}_{98} \mathrm{H}_{86} \mathrm{O}_{32}: \mathrm{C}, 66.28 ; \mathrm{H}, 4.88$. Found: C, 66.11; H, 5.02\%.
4.12. $2,3,4,6$-Tetra- $O$-benzoyl- $\alpha$-D-mannopyranosyl- $(1 \rightarrow$ 3)-[2- $O$-acetyl-3,4,6-tri- $O$-benzoyl- $\alpha$-D-mannopyranosyl$(1 \rightarrow 2)-3,4,6$-tri- $O$-benzoyl- $\alpha$-D-mannopyranosyl- $(1 \rightarrow 6)]-$ 2,4-di- $O$-acetyl- $\alpha$-D-mannopyranosyl trichloroacetimidate 19

Compound 16 ( $2.50 \mathrm{~g}, 1.41 \mathrm{mmol})$ was dissolved in 30 $\mathrm{mL} 90 \%$ TFA and stirred for $2 \mathrm{~h}$, and then the reaction mixture was poured directly into toluene $(150 \mathrm{~mL})$ and concentrated. Drying the residue under high vacuum gave a white foamy solid. This foamy solid was dissolved in pyridine $(20 \mathrm{~mL})$ and $\mathrm{Ac}_{2} \mathrm{O}(10 \mathrm{~mL})$ was added. The reaction mixture was stirred at $\mathrm{rt}$ for $12 \mathrm{~h}$, TLC (4:1 petroleum ether-EtOAc) indicated that the reaction was complete. The reaction mixture was concentrated to dryness. The resultant crude product $\mathbf{1 7}$ was dissolved in a $1 \mathrm{M}$ solution of ammonia-methanol $(100 \mathrm{~mL})$ and stirred at $\mathrm{rt}$ for $3 \mathrm{~h}$, at the end of which time TLC (3:1 petroleum ether-EtOAc) indicated that the reaction was complete. The solution was concentrated to give compound $\mathbf{1 8}$ as a syrup. A mixture of 18 , trichloroacetonitrile $(1.1 \mathrm{~mL}, 5 \mathrm{mmol})$, and 1,8 diazabicyclo[5.4.0]undecene (DBU) $(0.10 \mathrm{~mL}, \quad 0.80$ $\mathrm{mmol})$ in dry dichloromethane $(10 \mathrm{~mL})$ was stirred under nitrogen for $3 \mathrm{~h}$ and then concentrated. Purification of the residue by flash chromatography (3:1 petroleum ether-EtOAc) gave $\mathbf{1 9}$ as a white foam (2.34 $\mathrm{g}, 84 \%$ for four steps): $[\alpha]_{\mathrm{D}}+3.6\left(c 1.1, \mathrm{CHCl}_{3}\right) ;{ }^{1} \mathrm{H}$ NMR $\left(400 \mathrm{MHz}, \mathrm{CDCl}_{3}\right) \delta 9.04\left(\mathrm{~s}, 1 \mathrm{H}, \mathrm{CNH} \mathrm{CCl}_{3}\right)$, 8.11-7.27 (m, 50H, 10Ph), $6.33\left(\mathrm{~d}, 1 \mathrm{H}, J_{1,2}=0.8, \mathrm{H}-1\right)$, $6.22\left(\mathrm{dd}, 1 \mathrm{H}, J_{3,4}=J_{4,5}=10.0, \mathrm{H}-4\right), 5.99\left(\mathrm{dd}, 1 \mathrm{H}, J_{3,4}=\right.$ $\left.J_{4.5}=9.7, \mathrm{H}-4\right), 5.90-5.79(\mathrm{~m}, 4 \mathrm{H}, \mathrm{H}-2,3 \mathrm{H}-3$ and $\mathrm{H}-4)$, $5.68\left(\mathrm{dd}, 1 \mathrm{H}, J_{1,2}=0.8, J_{2,3}=2.6, \mathrm{H}-2\right), 5.64(\mathrm{dd}, 1 \mathrm{H}$, $\left.J_{1,2}=1.0, J_{2,3}=3.1, \mathrm{H}-2\right), 5.52-5.48(\mathrm{~m}, 2 \mathrm{H}, \mathrm{H}-2$ and $\mathrm{H}-4), 5.42\left(\mathrm{~d}, 1 \mathrm{H}, J_{1,2}=1.0, \mathrm{H}-1\right), 5.17\left(\mathrm{~d}, 1 \mathrm{H}, J_{1,2}=0.9\right.$, $\mathrm{H}-1), 5.09\left(\mathrm{~d}, 1 \mathrm{H}, J_{1,2}=1.1, \mathrm{H}-1\right), 4.65-4.49(\mathrm{~m}, 10 \mathrm{H})$, $4.33\left(\mathrm{dd}, 1 \mathrm{H}, J_{1,2}=1.0, J_{2,3}=2.9, \mathrm{H}-2\right), 4.19(\mathrm{ddd}, 1 \mathrm{H}$, $\left.J_{4,5}=9.8, J_{5,6}=4.7, J_{5,6^{\prime}}=2.0, \mathrm{H}-5\right), 3.93\left(\mathrm{dd}, 1 \mathrm{H}, J_{5,6}=\right.$ $\left.4.3, J_{6,6}=11.2, \mathrm{H}-6\right), 3.61\left(\mathrm{dd}, 1 \mathrm{H}, J_{5,6^{\prime}}=2.3, J_{6,6^{\prime}}=11.2\right.$, H-6'), 2.35 (s, 3H, MeCO), 2.29 (s, 3H, Me CO), 2.22 (s, $3 \mathrm{H}, \mathrm{MeCO}$ ). Anal. calcd for $\mathrm{C}_{102} \mathrm{H}_{88} \mathrm{Cl}_{3} \mathrm{NO}_{34}$ : C, 61.93; $\mathrm{H}, 4.48$. Found: $\mathrm{C}, 61.71 ; \mathrm{H}, 4.41 \%$.

4.13. $2,3,4,6$-Tetra- $O$-benzoyl- $\alpha$-D-mannopyranosyl-( $1 \rightarrow$ $3)-\{2,3,4,6$-tetra- $O$-benzoyl- $\alpha$-D-mannopyranosyl-( $1 \rightarrow 3)$ $[2,3,4,6$-tetra- $O$-benzoyl- $\alpha$-D-mannopyranosyl-(1 $\rightarrow 6)]-2,4-d i-O$-acetyl- $\alpha$-D-mannopyranosyl-(1 $\rightarrow 6)\}-1,2-$ $O$-ethylidene- $\beta$-D-mannopyranose 20

To a cooled solution $\left(0^{\circ} \mathrm{C}\right)$ of $5(784 \mathrm{mg}, 1 \mathrm{mmol})$ and $15(1.56 \mathrm{~g}, 1 \mathrm{mmol})$ in anhydrous $\mathrm{CH}_{2} \mathrm{Cl}_{2}(25 \mathrm{~mL})$ was added TMSOTf $(25 \mu \mathrm{L}, 0.14 \mathrm{mmol})$. The mixture was stirred at this temperature for $2 \mathrm{~h}$, and then quenched with $\mathrm{Et}_{3} \mathrm{~N}$ (two drops). The solvents were evaporated in vacuo to give a residue, which was purified by silica gel column chromatography (1:1 petroleum ether-EtOAc) to give pentasaccharide 20 as a syrup $(1.86 \mathrm{~g}, 85 \%)$. For $(R)$-isomer: $[\alpha]_{\mathrm{D}}^{25}-44.3\left(c 1.0, \mathrm{CHCl}_{3}\right) ;{ }^{1} \mathrm{H}$ NMR $(400$ $\left.\mathrm{MHz}, \mathrm{CDCl}_{3}\right) \delta 8.05-7.04(\mathrm{~m}, 60 \mathrm{H}, 12 \mathrm{Ph}), 6.17(\mathrm{dd}$, $\left.1 \mathrm{H}, J_{3,4}=J_{4,5}=10.0, \mathrm{H}-4\right), 6.03\left(\mathrm{dd}, 1 \mathrm{H}, J_{3,4}=J_{4,5}=\right.$ $10.0, \mathrm{H}-4), 6.00\left(\mathrm{dd}, 1 \mathrm{H}, J_{3,4}=J_{4,5}=10.4, \mathrm{H}-4\right), 5.93$ $\left(\mathrm{dd}, 1 \mathrm{H}, J_{2,3}=3.5, J_{3,4}=10.0, \mathrm{H}-3\right), 5.87\left(\mathrm{dd}, 1 \mathrm{H}, J_{2,3}=\right.$ 3.0, $\left.J_{3,4}=10.0, \mathrm{H}-3\right), 5.75-5.72(\mathrm{~m}, 2 \mathrm{H}, \mathrm{H}-2$ and $\mathrm{H}-3)$, 
$5.66\left(\mathrm{dd}, 1 \mathrm{H}, J_{1,2}=1.3, J_{2,3}=3.2, \mathrm{H}-2\right), 5.48(\mathrm{dd}, 1 \mathrm{H}$, $\left.J_{1,2}=1.0, J_{2,3}=3.5, \mathrm{H}-2\right), 5.46\left(\mathrm{dd}, 1 \mathrm{H}, J_{1,2}=1.5, J_{2,3}=\right.$ 3.0, H-2), $5.38\left(\mathrm{~d}, 1 \mathrm{H}, J_{1,2}=1.3, \mathrm{H}-1\right), 5.32(\mathrm{dd}, 1 \mathrm{H}$, $\left.J_{3,4}=J_{4,5}=10.5, \mathrm{H}-4\right), 5.26(\mathrm{q}, 1 \mathrm{H}, J=4.5, \mathrm{MeCH})$, $5.22\left(\mathrm{~d}, 1 \mathrm{H}, J_{1,2}=0.9, \mathrm{H}-1\right), 5.20\left(\mathrm{~d}, 1 \mathrm{H}, J_{1,2}=1.0, \mathrm{H}-1\right)$, $5.13\left(\mathrm{~d}, 1 \mathrm{H}, J_{1,2}=0.8, \mathrm{H}-1\right), 5.10\left(\mathrm{~d}, 1 \mathrm{H}, J_{1,2}=1.0, \mathrm{H}-1\right)$, 4.68-4.62 (m, 3H), 4.57-4.50 (m, 3H), 4.45-4.36 (m, $4 \mathrm{H}), 4.29\left(\mathrm{dd}, 1 \mathrm{H}, J_{1,2}=2.0, J_{2,3}=2.9, \mathrm{H}-2\right), 4.19-4.09$ $(\mathrm{m}, 3 \mathrm{H}, \mathrm{H}-4$ and $2 \mathrm{H}-6), 3.97$ (ddd, $1 \mathrm{H}, J_{4,5}=9.8, J_{5,6}=$ $\left.4.9, J_{5,6^{\prime}}=2.4, \mathrm{H}-5\right), 3.85\left(\mathrm{dd}, 1 \mathrm{H}, J_{5,6}=5.2, J_{6,6}=11.3\right.$, H-6), 3.78 (dd, $\left.1 \mathrm{H}, J_{2,3}=2.9, J_{3,4}=9.5, \mathrm{H}-3\right), 3.76(\mathrm{dd}$, $\left.1 \mathrm{H}, J_{5,6^{\prime}}=1.9, J_{6,6^{\prime}}=11.9, \mathrm{H}-6^{\prime}\right), 3.48\left(\mathrm{ddd}, 1 \mathrm{H}, J_{4,5}=\right.$ 9.8, $\left.J_{5,6}=5.2, J_{5,6^{\prime}}=2.2, \mathrm{H}-5\right), 2.21(\mathrm{~s}, 3 \mathrm{H}, M e \mathrm{CO}), 2.20$ $(\mathrm{s}, 3 \mathrm{H}, M e \mathrm{CO}), 1.48(\mathrm{~d}, 1 \mathrm{H}, J=4.5, M e \mathrm{CH}) ;{ }^{13} \mathrm{C} \mathrm{NMR}$ $\left(100 \mathrm{MHz}, \mathrm{CDCl}_{3}\right) \delta 170.4,170.3$ (2C, $\left.2 \mathrm{MeCO}\right), 166.1$, $166.0,165.9,165.8,165.7,165.4,165.3,165.2,165.1$, $165.0, \quad 164.9, \quad 164.8 \quad(12 \mathrm{C}, \quad 12 \mathrm{PhCO}), \quad 133.2-128.1$ (PhCO), $104.6\left(\mathrm{CH}_{3} \mathrm{CH}\right), 100.1,98.7,97.2,96.9,96.6$ (5C, 5C-1), 81.6, 79.3, 74.4, 73.6, 70.6, 70.5, 70.4, 70.1, $69.9,69.8,69.4,69.2,68.8,68.6,66.7,66.6,66.2,66.0$, $65.8,62.8,62.7,62.3$ (25C, 5C-2-6, some signals overlapped), 21.9 ( $\mathrm{MeCO}), 20.9,20.7$ (2C, $\left.2 \mathrm{CH}_{3} \mathrm{CH}\right)$. Anal. calcd for $\mathrm{C}_{120} \mathrm{H}_{106} \mathrm{O}_{40}: \mathrm{C}, 65.86 ; \mathrm{H}, 4.88$. Found $\mathrm{C}$, $65.62 ; \mathrm{H}, 4.80 \%$.

4.14. $\alpha$-D-Mannopyranosyl-( $(1 \rightarrow 3)-\{\alpha-D-m a n n o p y r a n o s y l-$ $(1 \rightarrow 3)$-[ $\alpha$-D-mannopyranosyl-(1 $\rightarrow 6)]-\alpha$-D-mannopyranosyl-(1 $\rightarrow 6)\}$ - $\alpha$-D-mannopyranose 22

Compound 20 (1.00 g, $0.457 \mathrm{mmol}$ ) was dissolved in $90 \%$ TFA $(15 \mathrm{~mL})$ and stirred for $2 \mathrm{~h}$, and then the reaction mixture was poured directly into toluene $(50 \mathrm{~mL})$ and concentrated. The residue was purified by flash chromatography (1:2 petroleum ether-EtOAc) to give $\mathbf{2 1}$ as a white foam $(0.931 \mathrm{~g}, 94 \%)$. Compound 21 was dissolved in a saturated ammonia-MeOH solution $(50 \mathrm{~mL})$. After 1 week at $\mathrm{rt}$, the reaction mixture was concentrated and the residue was purified by chromatography on Sephadex $\mathrm{LH}-20(\mathrm{MeOH})$ to afford $22(288 \mathrm{mg}, 76 \%)$ as a syrup with predominant $\alpha$-anomer at the reducing end; $[\alpha]_{\mathrm{D}}^{25}$ +46.5 ( $c$ 1.0, $\left.\mathrm{D}_{2} \mathrm{O}\right) ;{ }^{1} \mathrm{H}$ NMR (400 MHz, $\left.\mathrm{D}_{2} \mathrm{O}\right) \delta 5.03$, $5.01,4.94,4.71,4.70\left(\mathrm{~d}, 5 \mathrm{H}, J_{1,2} \approx 0,5 \mathrm{H}-1\right), 4.13-3.50(\mathrm{~m}$, $30 \mathrm{H}, 5 \mathrm{H}-2-6) ;{ }^{13} \mathrm{C}$ NMR (100 MHz, D $\left.2 \mathrm{O}\right) \delta 105.0,104.8$, 102.1, 101.9, 96.5 (5C, 5C-1); MALDI-TOF MS calcd for $\mathrm{C}_{30} \mathrm{H}_{52} \mathrm{O}_{26}: 828.72[\mathrm{M}]$. Found: $851.55[\mathrm{M}+\mathrm{Na}]$.

4.15. 2-O-Acetyl-3,4,6-tri- $O$-benzoyl- $\alpha$-D-mannopyranosyl-( $1 \rightarrow 2)-3,4,6$-tri- $O$-benzoyl- $\alpha$-D-mannopyranosyl-(1 $\rightarrow 3)-\{2,3,4,6$-tetra- $O$-benzoyl- $\alpha$-D-mannopyranosyl-(1 $\rightarrow$ 3)-[2,3,4,6-tetra- $O$-benzoyl- $\alpha$-D-mannopyranosyl- $(1 \rightarrow 6)]-$ 2,4-di- $O$-acetyl- $\alpha$-D-mannopyranosyl- $(1 \rightarrow 6)\}-1,2-O$-ethylidene- $\beta$-D-mannopyranose 23

To a cooled solution $\left(0^{\circ} \mathrm{C}\right)$ of $\mathbf{8}(598 \mathrm{mg}, 0.5 \mathrm{mmol})$ and 15 (782 $\mathrm{mg}, 0.5 \mathrm{mmol})$ in anhydrous $\mathrm{CH}_{2} \mathrm{Cl}_{2}(25 \mathrm{~mL})$ was added TMSOTf $(9.0 \mu \mathrm{L}, 0.05 \mathrm{mmol})$. The mixture was stirred at this temperature for $2 \mathrm{~h}$, and then quenched with $\mathrm{Et}_{3} \mathrm{~N}$ (two drops). The solvents were evaporated in vacuo to give a residue, which was purified by silica gel column chromatography $(1: 1$ petroleum ether-EtOAc) to give hexaccharide $\mathbf{2 3}$ as a foamy solid (1.02 g, 79\%). For the $(R)$-isomer: $[\alpha]_{\mathrm{D}}^{25}-14.3\left(c 1.5, \mathrm{CHCl}_{3}\right) ;{ }^{1} \mathrm{H}$ NMR $\left(400 \mathrm{MHz} \mathrm{CDCl}_{3}\right) \delta 8.14-7.18(\mathrm{~m}, 70 \mathrm{H}, 14 \mathrm{Ph}), 6.24(\mathrm{dd}$, $\left.1 \mathrm{H}, J_{3,4}=J_{4,5}=9.8, \mathrm{H}-4\right), 6.13\left(\mathrm{dd}, 1 \mathrm{H}, J_{3,4}=J_{4,5}=10.2\right.$, H-4), 5.99 (dd, $\left.1 \mathrm{H}, J_{3,4}=J_{4,5}=10.1, \mathrm{H}-4\right), 5.96(\mathrm{dd}, 1 \mathrm{H}$, $\left.J_{2,3}=3.2, J_{3,4}=10.1, \mathrm{H}-3\right), 5.87\left(\mathrm{dd}, 1 \mathrm{H}, J_{2,3}=3.1, J_{3,4}=\right.$ 9.8, H-3), 5.89 (dd, $\left.1 \mathrm{H}, J_{3,4}=J_{4,5}=9.7, \mathrm{H}-4\right), 5.86(\mathrm{dd}$, $\left.1 \mathrm{H}, J_{1,2}=1.4, J_{2,3}=3.1, \mathrm{H}-2\right), 5.81\left(\mathrm{dd}, 1 \mathrm{H}, J_{2,3}=3.0\right.$, $\left.J_{3,4}=10.5, \mathrm{H}-3\right), 5.76\left(\mathrm{dd}, 1 \mathrm{H}, J_{1,2}=1.5, J_{2,3}=3.2, \mathrm{H}-2\right)$, $5.64\left(\mathrm{dd}, 1 \mathrm{H}, J_{1,2}=1.3, J_{2,3}=3.1, \mathrm{H}-2\right), 5.55-5.52(\mathrm{~m}, 3 \mathrm{H}$, $\mathrm{H}-1, \mathrm{H}-2$ and $\mathrm{H}-3), 5.44\left(\mathrm{dd}, 1 \mathrm{H}, J_{3,4}=J_{4,5}=9.6, \mathrm{H}-4\right)$, $5.41\left(\mathrm{~d}, 1 \mathrm{H}, J_{1,2}=1.0, \mathrm{H}-1\right), 5.28(\mathrm{q}, 1 \mathrm{H}, J=4.6, \mathrm{MeCH})$, $5.20\left(\mathrm{~d}, 1 \mathrm{H}, J_{1,2}=1.5, \mathrm{H}-1\right), 5.04\left(\mathrm{~d}, 1 \mathrm{H}, J_{1,2}=0.7, \mathrm{H}-1\right)$, $5.02\left(\mathrm{~d}, 1 \mathrm{H}, J_{1,2}=1.1, \mathrm{H}-1\right), 5.00\left(\mathrm{~d}, 1 \mathrm{H}, J_{1,2}=0.8, \mathrm{H}-1\right)$, 4.73-4.46 (m, 15H), 4.25-4.16 (m, 3H), 4.04 (ddd, $1 \mathrm{H}$, $\left.J_{4,5}=9.6, J_{5,6}=5.1, J_{5,6^{\prime}}=2.2, \mathrm{H}-5\right), 3.92\left(\mathrm{dd}, 1 \mathrm{H}, J_{5,6}=\right.$ $5.6, J_{6,6}=12.4$, H-6), $3.78\left(\mathrm{dd}, 1 \mathrm{H}, J_{2,3}=3.3, J_{3,4}=9.8\right.$, H-3), $3.70\left(\mathrm{dd}, 1 \mathrm{H}, J_{5,6^{\prime}}=2.3, J_{6,6^{\prime}}=12.4, \mathrm{H}-6^{\prime}\right), 3.38$ $\left(\mathrm{ddd}, 1 \mathrm{H}, J_{4,5}=9.7, J_{5,6}=5.1, J_{5,6^{\prime}}=2.3, \mathrm{H}-5\right), 2.30(\mathrm{~s}, 3 \mathrm{H}$, $M e \mathrm{CO}$ ), 2.26 (s, 3H, MeCO), 1.93 (s, 3H, MeCO), 1.52 $(\mathrm{d}, 1 \mathrm{H}, J=4.6, \mathrm{MeCH}) ;{ }^{13} \mathrm{C} \mathrm{NMR}\left(100 \mathrm{MHz}, \mathrm{CDCl}_{3}\right)$ $\delta 170.1,170.0,168.7(3 \mathrm{C}, 3 \mathrm{MeCO}), 165.8,165.7,165.6$, $165.5,165.3,165.1,165.0,164.9,164.8,164.6(14 \mathrm{C}$, 14PhCO, some signals overlapped), 133.1-127.9 (PhCO), $104.2\left(\mathrm{CH}_{3} \mathrm{CH}\right), 100.6,99.1,98.5,97.2,96.9,96.3(6 \mathrm{C}$, 6C-1), 80.5, 78.9, 74.4, 73.8, 70.6, 70.4, 70.2, 70.0, 69.2, $69.0,68.9,68.8,68.5,68.1,67.3,67.0,66.4,66.0,65.7$, $63.4,63.0,62.5,61.9(30 \mathrm{C}, 6 \mathrm{C}-2-6$, some signals overlapped), $21.6\left(\mathrm{CH}_{3} \mathrm{CH}\right), 20.5,20.3,20.1$ (3C, 3Me CO). Anal. calcd for $\mathrm{C}_{142} \mathrm{H}_{126} \mathrm{O}_{48}: \mathrm{C}, 65.58 ; \mathrm{H}, 4.88$. Found $\mathrm{C}$, $65.78 ; \mathrm{H}, 4.75 \%$.

4.16. $\alpha$-D-Mannopyranosyl-( $1 \rightarrow 2)$ - $\alpha$-D-mannopyranosyl$(1 \rightarrow 3)$ - $\{\alpha$-D-mannopyranosyl- $(1 \rightarrow 3)$-[ $\alpha$-D-mannopyranosyl$(1 \rightarrow 6) \mid-\alpha$-D-mannopyranosyl- $(1 \rightarrow 6)\}-\alpha$-D-mannopyranose 25

Compound 23 (910 mg, $0.35 \mathrm{mmol}$ ) was dissolved in $90 \%$ TFA $(15 \mathrm{~mL})$ and stirred for $2 \mathrm{~h}$, and then the reaction mixture was poured directly into toluene $(50 \mathrm{~mL})$ and concentrated. The residue was purified by flash chromatography (1:2 petroleum ether-EtOAc) to give $\mathbf{2 4}$ as a foamy solid $(730 \mathrm{mg}, 81 \%)$. Compound $\mathbf{2 4}$ was dissolved in a saturated ammonia-MeOH solution $(50 \mathrm{~mL})$. After 1 week at $\mathrm{rt}$, the reaction mixture was concentrated and the residue was purified by chromatography on Sephadex LH-20 (MeOH) to afford 25 as a syrup (203 $\mathrm{mg}, 72 \%$ ) with predominant $\alpha$-anomer at the reducing end: $[\alpha]_{\mathrm{D}}^{25}+80.0\left(c 1.0, \mathrm{D}_{2} \mathrm{O}\right) ;{ }^{1} \mathrm{H} \mathrm{NMR}\left(400 \mathrm{MHz}, \mathrm{D}_{2} \mathrm{O}\right)$ $\delta 5.24,5.03,4.93,4.78,4.73,4.71\left(\mathrm{~d}, 6 \mathrm{H}, J_{1,2} \approx 0,6 \mathrm{H}-1\right)$, 4.13-3.50 (m, 36H, 6H-2-6); ${ }^{13} \mathrm{C}$ NMR (100 MHz, D $\left.2 \mathrm{O}\right)$ $\delta$ 104.7, 104.6, 103.0, 102.0, 101.8, 101.7 (6C, 6C-1). MALDI-TOF MS calcd for $\mathrm{C}_{36} \mathrm{H}_{62} \mathrm{O}_{31}$ : 990.86 [M]. Found: $1013.74[\mathrm{M}+\mathrm{Na}]$.

4.17. $2,3,4,6$-Tetra- $O$-benzoyl- $\alpha$-D-mannopyranosyl-( $1 \rightarrow$ 2)-3,4,6-tri- $O$-benzoyl- $\alpha$-D-mannopyranosyl- $(1 \rightarrow 2)-3,4,6-$ tri- $O$-benzoyl- $\alpha$-D-mannopyranosyl- $(1 \rightarrow 3)-\{2,3,4,6-$ tetra- $O$-benzoyl- $\alpha$-D-mannopyranosyl- $(1 \rightarrow 3)-[2,3,4,6-$ tetra- $O$-benzoyl- $\alpha$-D-mannopyranosyl-( $1 \rightarrow 6) \mid-2,4-d i-O$ acetyl- $\alpha-D-m a n n o p y r a n o s y l-(1 \rightarrow 6)\}-1,2-O$-ethylidene$\beta$-D-mannopyranose 26

To a cooled solution $\left(0^{\circ} \mathrm{C}\right)$ of $\mathbf{1 1}(866 \mathrm{mg}, 0.5 \mathrm{mmol})$ and 15 (782 mg, $0.5 \mathrm{mmol})$ in anhydrous $\mathrm{CH}_{2} \mathrm{Cl}_{2}(25 \mathrm{~mL})$ was added TMSOTf $(9 \mu \mathrm{L}, 0.05 \mathrm{mmol})$. The mixture was 
stirred at this temperature for $2 \mathrm{~h}$, and then quenched with $\mathrm{Et}_{3} \mathrm{~N}$ (two drops). Evaporation of the solvents in vacuo gave a residue, and purification of the residue by silica gel column chromatography (1:1 petroleum etherEtOAc) gave heptasaccharide $\mathbf{2 6}$ as a white foam (1.29 g, $82 \%)$. For the $(S)$-isomer: $[\alpha]_{\mathrm{D}}^{25}-17.5\left(c 1.0, \mathrm{CHCl}_{3}\right)$; ${ }^{1} \mathrm{H}$ NMR $\left(400 \mathrm{MHz}, \mathrm{CDCl}_{3}\right) \delta 8.05-7.22(\mathrm{~m}, 90 \mathrm{H}$, $18 \mathrm{Ph}), 6.22\left(\mathrm{dd}, 1 \mathrm{H}, J_{3,4}=J_{4,5}=10.1, \mathrm{H}-4\right), 6.14(\mathrm{dd}$, $\left.1 \mathrm{H}, J_{3,4}=J_{4,5}=10.0, \mathrm{H}-4\right), 6.06-5.89(\mathrm{~m}, 6 \mathrm{H}), 5.82-5.76$ $(\mathrm{m}, 4 \mathrm{H}), 5.66(\mathrm{q}, 1 \mathrm{H}, J=4.9, \mathrm{MeCH}), 5.54-5.52(\mathrm{~m}$, $2 \mathrm{H}, 2 \mathrm{H}-2), 5.02\left(\mathrm{dd}, 1 \mathrm{H}, J_{3,4}=J_{4,5}=9.9, \mathrm{H}-4\right), 5.47-$ $5.46\left(2 \mathrm{~d}, 2 \mathrm{H}, J_{1,2}=1.4,2 \mathrm{H}-2\right), 5.36\left(\mathrm{~d}, 1 \mathrm{H}, J_{1,2}=1.3\right.$, $\mathrm{H}-1), 5.23\left(\mathrm{~d}, 1 \mathrm{H}, J_{1,2}=1.5, \mathrm{H}-1\right), 5.16\left(\mathrm{~d}, 1 \mathrm{H}, J_{1,2}=1.0\right.$, $\mathrm{H}-1), 5.02\left(\mathrm{~d}, 1 \mathrm{H}, J_{1,2}=1.3, \mathrm{H}-1\right), 4.98\left(\mathrm{~d}, 1 \mathrm{H}, J_{1,2}=1.0\right.$, $\mathrm{H}-1), 4.73-4.39(\mathrm{~m}, 18 \mathrm{H}), 4.19-3.96(\mathrm{~m}, 6 \mathrm{H}), 3.78(\mathrm{dd}$, $\left.1 \mathrm{H}, J_{2,3}=3.4, J_{3,4}=9.4, \mathrm{H}-3\right), 3.70\left(\mathrm{dd}, 1 \mathrm{H}, J_{5,6^{\prime}}=2.2\right.$, $\left.J_{6,6^{\prime}}=11.3, \mathrm{H}-6^{\prime}\right), 3.38\left(\mathrm{ddd}, 1 \mathrm{H}, J_{4.5}=9.4, J_{5,6}=5.5\right.$, $\left.J_{5,6^{\prime}}=2.2, \mathrm{H}-5\right), 2.35$ (s, 3H, MeCO), 2.24 (s, 3H, $M e \mathrm{CO}), 1.32(\mathrm{~d}, 1 \mathrm{H}, J=4.9, M e \mathrm{CH}) ;{ }^{13} \mathrm{C}$ NMR $(100$ $\left.\mathrm{MHz}, \mathrm{CDCl}_{3}\right) \delta 170.8,170.4(2 \mathrm{C}, 2 \mathrm{MeCO}), 166.6$, $166.4,166.3,166.2,166.1,165.6,165.5,165.4,165.1$, 164.8 (18C, $18 \mathrm{PhCO}$, some signals overlapped), 133.5128.4 (PhCO), 103.8 $\left(\mathrm{CH}_{3} \mathrm{CH}\right), 101.3,99.7,99.7,99.1$, 97.7, 97.6, 97.5 (7C, 7C-1), 81.5, 75.3, 74.4, 71.0, 70.8, $70.5,70.3,70.1,69.9,69.6,69.2,69.0,68.3,67.9,66.9$, $66.7,66.4,66.1,64.0,63.5,63.0,62.6,62.5(35 \mathrm{C}, 7 \mathrm{C}-2-$ 6, some signals overlapped), $21.2\left(\mathrm{CH}_{3} \mathrm{CH}\right), 21.0,20.8$ $(2 \mathrm{C}, 2 \mathrm{Me} \mathrm{CO})$. Anal. calcd for $\mathrm{C}_{174} \mathrm{H}_{150} \mathrm{O}_{56}: \mathrm{C}, 66.62$; $\mathrm{H}, 4.82$. Found $\mathrm{C}, 66.84 ; \mathrm{H}, 4.77 \%$.

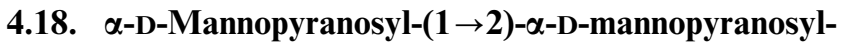
$(1 \rightarrow 2)-\alpha$-D-mannopyranosyl- $(1 \rightarrow 3)-\{\alpha-D-m a n n o p y r a n o-$ syl-(1 $\rightarrow 3)$-[ $\alpha$-D-mannopyranosyl-( $1 \rightarrow 6)]-\alpha$-D-mannopyranosyl- $(1 \rightarrow 6)\}-\alpha$-D-mannopyranose 28

Compound 26 ( $1.00 \mathrm{~g}, 0.32 \mathrm{mmol})$ was dissolved in $90 \%$ TFA $(15 \mathrm{~mL})$ and stirred for $2 \mathrm{~h}$, and then the reaction mixture was poured directly into toluene $(50 \mathrm{~mL})$ and concentrated. The residue was purified by flash chromatography (1:2 petroleum ether-EtOAc) to give 27 (860 $\mathrm{mg}, 87 \%$ ) as a white foam. Compound 27 was dissolved in a saturated ammonia-MeOH solution $(50 \mathrm{~mL})$. After 1 week at $\mathrm{rt}$, the reaction mixture was concentrated and the residue was purified by chromatography on Sephadex $\mathrm{LH}-20(\mathrm{MeOH})$ to afford $28(242 \mathrm{mg}, 76 \%)$ as a foamy solid with predominant $\alpha$-anomer at the reducing end: $[\alpha]_{\mathrm{D}}^{25}+74.5\left(c 1.0, \mathrm{D}_{2} \mathrm{O}\right) ;{ }^{1} \mathrm{H}$ NMR $\left(400 \mathrm{MHz}, \mathrm{D}_{2} \mathrm{O}\right) \delta$ $5.22,5.17,5.01,5.00,4.91,4.77,4.72\left(\mathrm{~d}, 7 \mathrm{H}, J_{1.2} \approx 0\right.$, $7 \mathrm{H}-1), 4.02-3.50(\mathrm{~m}, 42 \mathrm{H}, 7 \mathrm{H}-2-6) ;{ }^{13} \mathrm{C}$ NMR $(100 \mathrm{MHz}$, $\left.\mathrm{D}_{2} \mathrm{O}\right) \delta 103.4,103.1,103.0,102.9,102.1,101.9,101.9(7 \mathrm{C}$, 7C-1). MALDI-TOF MS calcd for $\mathrm{C}_{42} \mathrm{H}_{72} \mathrm{O}_{36}: 1152.99$ [M]. Found: $1175.87[\mathrm{M}+\mathrm{Na}]$.

4.19. $2,3,4,6$-Tetra- $O$-benzoyl- $\alpha$-D-mannopyranosyl-( $1 \rightarrow$ 2)-3,4,6-tri- $O$-benzoyl- $\alpha$-D-mannopyranosyl- $(1 \rightarrow 2)-3,4,6-$ tri- $O$-benzoyl- $\alpha$-D-mannopyranosyl- $(1 \rightarrow 3)-\{2,3,4,6$-tetra$O$-benzoyl- $\alpha$-D-mannopyranosyl- $(1 \rightarrow 3)$-[2- $O$-acetyl3,4,6-tri- $O$-benzoyl- $\alpha$-D-mannopyranosyl-( $1 \rightarrow 2)-3,4,6-$ tri- $O$-benzoyl- $\alpha$-D-mannopyranosyl- $(1 \rightarrow 6) \mid-2,4$-di$O$-acetyl- $\alpha$-D-mannopyranosyl- $(1 \rightarrow 6)\}-1,2-O$-ethylidene$\beta$-D-mannopyranose 29

To a cooled solution $\left(0^{\circ} \mathrm{C}\right)$ of $\mathbf{1 1}(433 \mathrm{mg}, 0.25 \mathrm{mmol})$ and 19 (494 mg, $0.25 \mathrm{mmol})$ in anhydrous $\mathrm{CH}_{2} \mathrm{Cl}_{2}(25$ $\mathrm{mL})$ was added TMSOTf $(9 \mu \mathrm{L}, 0.05 \mathrm{mmol})$. The mixture was stirred at this temperature for $2 \mathrm{~h}$, and then quenched with $\mathrm{Et}_{3} \mathrm{~N}$ (two drops). The solvents were evaporated in vacuo to give a residue, which was purified by silica gel column chromatography (1:1 petroleum ether-EtOAc) to give octasaccharide $\mathbf{2 9}$ as a syrup $(710 \mathrm{mg}, 80 \%)$. For the $(R)$-isomer: $[\alpha]_{\mathrm{D}}^{25}-9.9(c$ 1.0, $\mathrm{CHCl}_{3}$ ); ${ }^{1} \mathrm{H}$ NMR (400 MHz, $\left.\mathrm{CDCl}_{3}\right) \delta 8.05-7.22$ $(\mathrm{m}, 100 \mathrm{H}, 20 \mathrm{Ph}), 6.21\left(\mathrm{dd}, 1 \mathrm{H}, J_{3,4}=J_{4,5}=10.0, \mathrm{H}-4\right)$, 6.04-5.90 (m, 9H), 5.81-5.78 (m, 3H), $5.65(\mathrm{dd}, 1 \mathrm{H}$, $\left.J_{1,2}=1.1, J_{2,3}=3.2, \mathrm{H}-2\right), 5.53-5.50(\mathrm{~m}, 3 \mathrm{H}), 5.46(\mathrm{~d}$, $\left.1 \mathrm{H}, J_{1,2}=1.2, \mathrm{H}-1\right), 5.43\left(\mathrm{~d}, 1 \mathrm{H}, J_{1,2}=1.5, \mathrm{H}-1\right), 5.37$ (d, $\left.1 \mathrm{H}, J_{1,2}=1.2, \mathrm{H}-1\right), 5.27$ (q, $1 \mathrm{H}, J=4.8, \mathrm{MeC} H$ ), $5.25\left(\mathrm{~d}, 1 \mathrm{H}, J_{1,2}=1.3, \mathrm{H}-1\right), 5.14\left(\mathrm{~d}, 1 \mathrm{H}, J_{1,2}=1.5\right.$, $\mathrm{H}-1), 5.07\left(\mathrm{~d}, 1 \mathrm{H}, J_{1,2}=1.4, \mathrm{H}-1\right), 5.01\left(\mathrm{~d}, 1 \mathrm{H}, J_{1,2}=\right.$ $1.4, \mathrm{H}-1), 4.97\left(\mathrm{~d}, 1 \mathrm{H}, J_{1,2}=1.2, \mathrm{H}-1\right), 4.70-4.26(\mathrm{~m}$, $21 \mathrm{H}), 4.18-4.08(\mathrm{~m}, 5 \mathrm{H}), 3.98-3.92(\mathrm{~m}, 2 \mathrm{H}), 3.88(\mathrm{dd}$, $\left.1 \mathrm{H}, J_{2,3}=3.2, J_{3,4}=9.6, \mathrm{H}-3\right), 3.57\left(\mathrm{dd}, 1 \mathrm{H}, J_{5,6^{\prime}}=2.0\right.$, $\left.J_{6,6^{\prime}}=10.8, \mathrm{H}-6^{\prime}\right), 3.42\left(\mathrm{ddd}, 1 \mathrm{H}, J_{4,5}=9.8, J_{5,6}=4.9\right.$, $\left.J_{5,6^{\prime}}=2.1, \mathrm{H}-5\right), 2.21(\mathrm{~s}, 3 \mathrm{H}, M e \mathrm{CO}), 2.18(\mathrm{~s}, 3 \mathrm{H}$, $M e \mathrm{CO}), 1.95(\mathrm{~s}, 3 \mathrm{H}, M e \mathrm{CO}), 1.48(\mathrm{~d}, 1 \mathrm{H}, J=4.8$, $\mathrm{MeCH}) ;{ }^{13} \mathrm{C}$ NMR $\left(100 \mathrm{MHz}, \mathrm{CDCl}_{3}\right) \delta 170.9,169.8$, 167.4 (3C, 3MeCO), 165.6-164.6 ( $\mathrm{PhCO}$ ), 133.0-128.0 (PhCO), 104.1 $\left(\mathrm{CH}_{3} \mathrm{CH}\right), 100.8,99.5,99.3,99.2,98.5$, 97.6, 97.2, 96.2 (7C, 7C-1), 81.6-61.9 (C-2-6), 21.6 $\left(\mathrm{CH}_{3} \mathrm{CH}\right), 20.5,20.4,20.1$ (3C, 3Me CO). Anal. calcd for $\mathrm{C}_{196} \mathrm{H}_{170} \mathrm{O}_{64}: \mathrm{C}, 66.32 ; \mathrm{H}, 4.83$. Found $\mathrm{C}, 66.55 ; \mathrm{H}$, $4.88 \%$.

4.20. $\alpha$-D-Mannopyranosyl-(1 $\rightarrow 2)-\alpha$-D-mannopyranosyl$(1 \rightarrow 2)-\alpha$-D-mannopyranosyl-( $1 \rightarrow 3)$ - $\{\alpha-D-m a n n o p y r a n o-$ syl-( $1 \rightarrow 3)-[\alpha$-D-mannopyranosyl-( $1 \rightarrow 2)-\alpha$-D-mannopyranosyl-( $(1 \rightarrow 6) \mid-\alpha$-D-mannopyranosyl- $(1 \rightarrow 6)\}-\alpha$-Dmannopyranose 31

Compound 29 (600 mg, $0.17 \mathrm{mmol})$ was dissolved in $90 \%$ TFA (15 mL) and stirred for $2 \mathrm{~h}$, and then the reaction mixture was poured directly into toluene (50 $\mathrm{mL}$ ) and concentrated. The residue was purified by flash chromatography (1:2 petroleum ether-EtOAc) to give $30(510 \mathrm{mg}, 86 \%)$ as a white foam. Compound 30 was dissolved in a saturated ammonia- $\mathrm{MeOH}$ solution $(50 \mathrm{~mL})$. After 1 week at $\mathrm{rt}$, the reaction mixture was concentrated and the residue was purified by chromatography on Sephadex LH-20 (MeOH) to afford 31 $(154 \mathrm{mg}, 82 \%)$ as a syrup with predominant $\alpha$-anomer at the reducing end: $[\alpha]_{\mathrm{D}}^{25}+30.7\left(c 1.0, \mathrm{D}_{2} \mathrm{O}\right) ;{ }^{1} \mathrm{H}$ NMR $\left(400 \mathrm{MHz}, \mathrm{D}_{2} \mathrm{O}\right) \delta 5.43,5.39,5.34,5.27,5.11,5.08$, $5.02,5.00\left(\mathrm{~d}, 8 \mathrm{H}, J_{1,2} \approx 0,8 \mathrm{H}-1\right), 4.11-3.31(\mathrm{~m}, 48 \mathrm{H}$, $8 \mathrm{H}-2-6) ;{ }^{13} \mathrm{C}$ NMR $\left(100 \mathrm{MHz}, \mathrm{D}_{2} \mathrm{O}\right) \delta 105.4,105.3$, $105.0,104.8,103.9,101.8,101.4,100.9$ (8C, 8C-1). MALDI-TOF MS calcd for $\mathrm{C}_{48} \mathrm{H}_{82} \mathrm{O}_{41}: 1315.14$ [M]. Found: $1338.27[\mathrm{M}+\mathrm{Na}]$.

\section{Acknowledgements}

This work was supported by CAS KIP-RCEES9904, NNSF of China (Projects 39970864, and 30070815), and the Ministry of Science and Technology. 


\section{References}

1. Essentials of Glycobiology, Varki, A.; Cummings, R.; Esko, J.; Freeze, H.; Hart, G.; Marth, J., Eds.; Cold Spring Harbor Laboratory Press: Cold Spring Harbor, New York, 1999.

2. (a) Wyss, D. F.; Choi, J. S.; Li, J.; Knoppers, M. H.; Willis, K. J.; Arulanandam, A. R. N.; Smolyar, A.; Reinherz, E. L.; Wagner, G. Science 1995, 269, 1273; (b) Recny, M. A.; Luther, M. A.; Knoppers, M. H.; Neidhardt, E. A.; Khandekar, S. S.; Concino, M. F.; Shimke, P. A.; Francis, M. A.; Moebius, U. J. Biol. Chem. 1992, 267, 22428.

3. (a) Bewley, C. A.; Otero-Quintero, S. J. Am. Chem. Soc. 2001, 123, 3892; (b) Hansen, J.-E. S.; Nielsen, C.; Arendrup, M.; Olofsson, S.; Mathiesen, L.; Nielsen, J. O.; Clausen, H. J. Virol. 1991, 65, 6461; (c) Hansen, J.-E. S.; Clausen, H.; Sorensen, T.; White, T.; Wandall, H. H. Alfred Benzon Symp. 1994, 36, 297.

4. (a) Rademann, J.; Geyer, A.; Schmidt, R. R. Angew. Chem., Int. Ed. 1998, 37, 1241; (b) Ogawa, T.; Katano, K.; Sasajima, K.; Matsui, M. Tetrahedron 1981, 37, 2779; (c)
Ogawa, T.; Sasajima, K. Tetrahedron 1981, 37, 2787; (d) Guo, Z.; Nakahara, Y.; Ogawa, T. Tetrahedron Lett. 1997, 38, 4799; (e) Depre, D.; Duffels, A.; Green, L. G.; Lenz, R.; Ley, S. V.; Wong, C.-H. Chem. Eur. J. 1999, 5, 3326; (f) Dan, A.; Lergenmuller, M.; Amano, M.; Nakahara, Y.; Ogawa, T.; Ito, Y. Chem. Eur. J. 1998, 4, 2182; (g) Unverzagt, C.; Seifert, J. Tetrahedron Lett. 1997, 38, 7857; (h) Weiler, S.; Schmidt, R. R. Tetrahedron Lett. 1998, 39, 2292; (i) Wang, W.; Kong, F. Angew. Chem., Int. Ed. 1999, 38, 1247; (j) Becker, B.; Furneaux, R. H.; Reck, F.; Zubkov, O. A. Carbohydr. Res. 1999, 315, 148; (k) Ogawa, T.; Katano, K.; Matsui, M. Carbohydr. Res. 1978, 64, c3.

5. Bachhawat, K.; Thomas, C. J.; Amutha, B.; Krishnasastry, M. V.; Khan, M. I.; Surolia, A. J. Biol. Chem. 2001, 276, 5541.

6. (a) Zhu, Y.; Kong, F. Synlett 2000, 663; (b) Unverzagt, C. Angew. Chem., Int. Ed. Engl. 1994, 33, 1102.

7. Betaneli, V. I.; Ovchinnikov, M. V.; Kochetkov, N. K. Carbohydr. Res. 1982, 107, 285.

8. Zhu, Y.; Kong, F. Synlett 2000, 1783.

9. Zhu, Y.; Kong, F. Synlett 2001, 1217. 